\title{
Active Ageing
} A Policy Framework

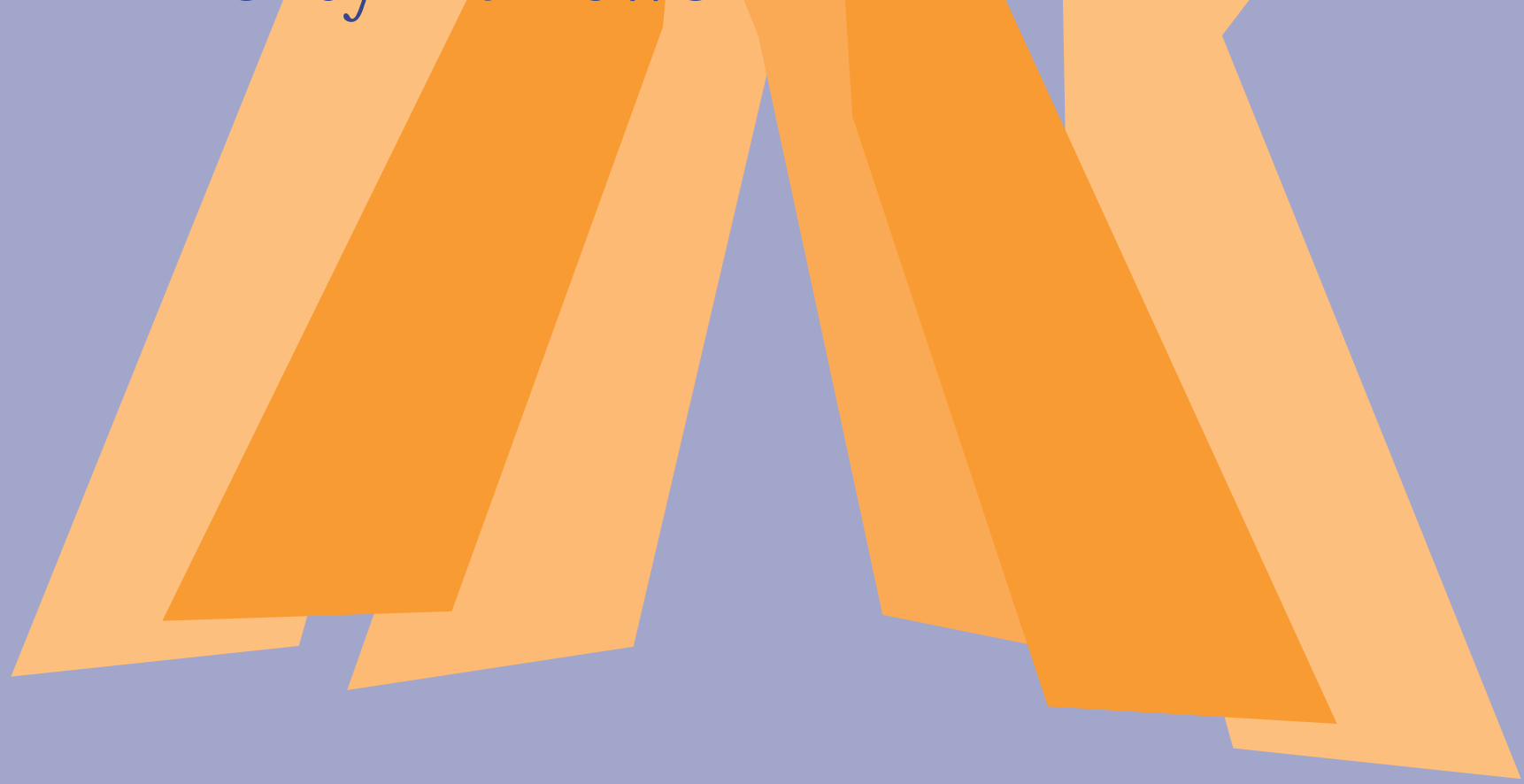




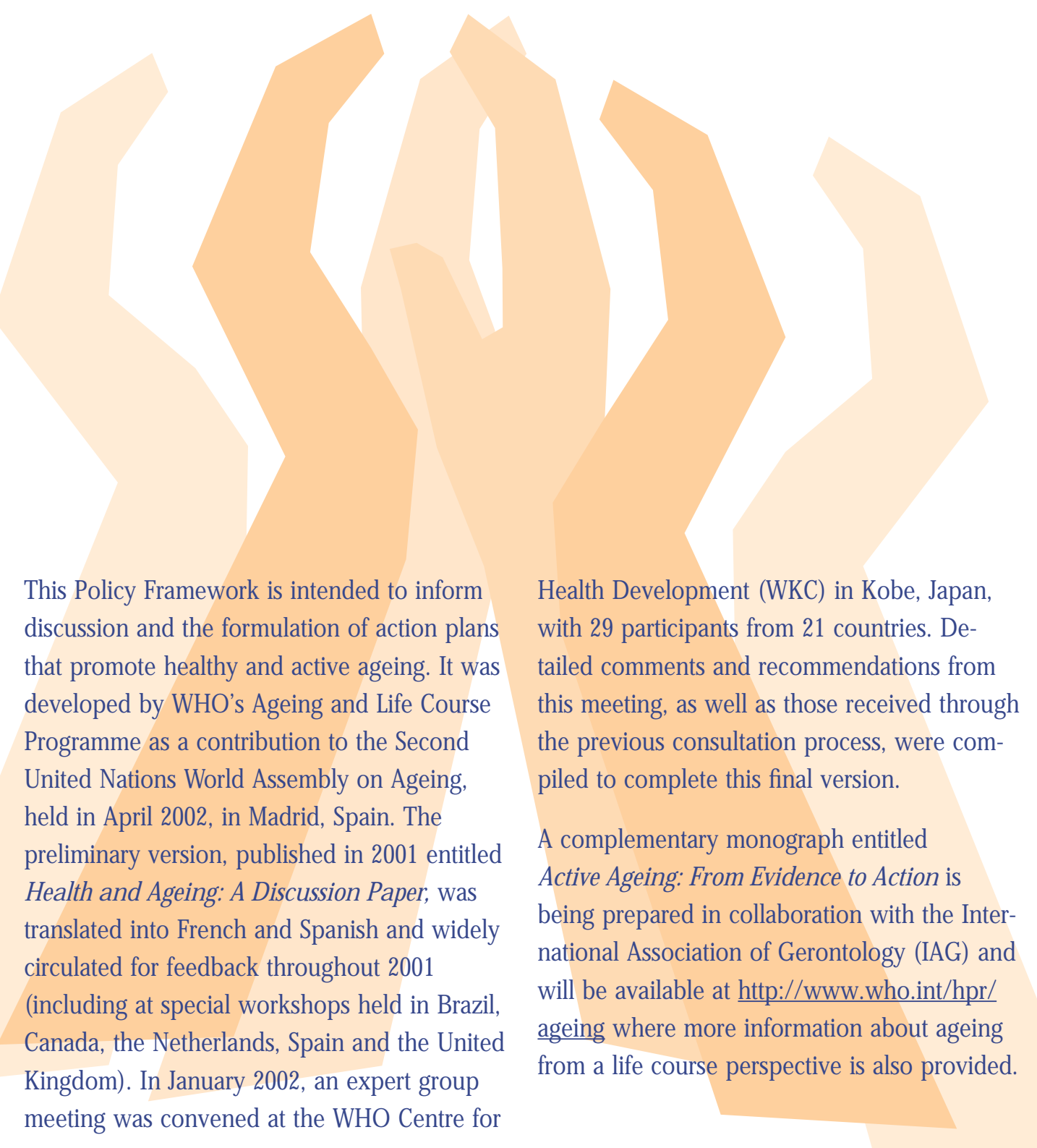

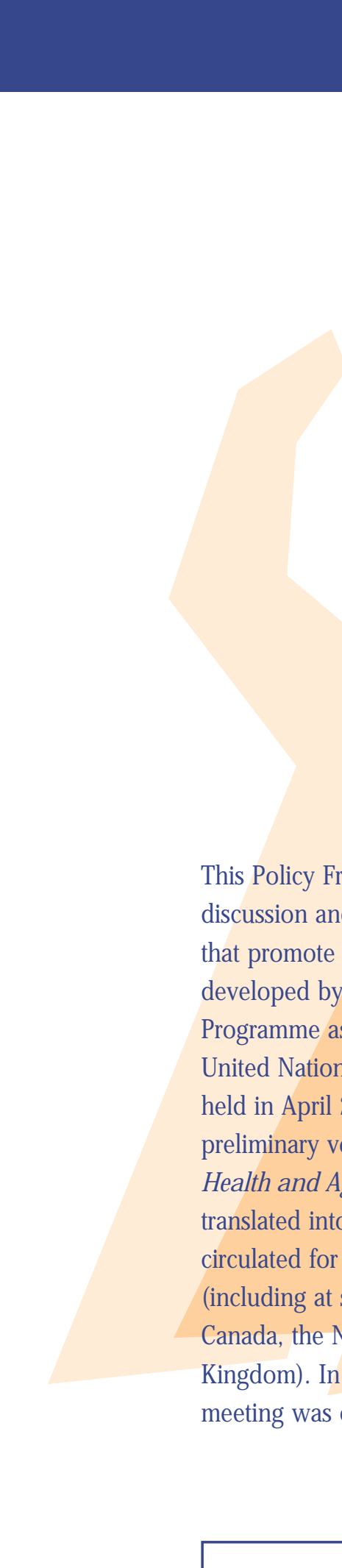

A contribution of the World Health Organization to the Second United Nations World Assembly on Ageing, Madrid, Spain, April 2002. 


\section{Contents}

$\begin{array}{ll}\text { Introduction } & 5\end{array}$

1. Global Ageing: A Triumph and a Challenge $\quad 6$

The Demographic Revolution 6

Rapid Population Ageing in Developing Countries $\quad 9$

2. Active Ageing: The Concept and Rationale $\quad 12$

What is "Active Ageing"? $\quad 12$

A Life Course Approach to Active Ageing $\quad 14$

Active Ageing Policies and Programmes 16

3. The Determinants of Active Ageing: Understanding the Evidence 19

Cross-Cutting Determinants: Culture and Gender 20

Determinants Related to Health and Social Service Systems 21

Behavioural Determinants $\quad 22$

Determinants Related to Personal Factors $\quad 26$

Determinants Related to the Physical Environment $\quad 27$

Determinants Related to the Social Environment $\quad 28$

Economic Determinants $\quad 30$

4. Challenges of an Ageing Population 33

Challenge 1: The Double Burden of Disease 33

Challenge 2: Increased Risk of Disability 34

Challenge 3: Providing Care for Ageing Populations 37

Challenge 4: The Feminization of Ageing $\quad 39$

Challenge 5: Ethics and Inequities $\quad 40$

Challenge 6: The Economics of an Ageing Population $\quad 42$

Challenge 7: Forging a New Paradigm 43

5. The Policy Response $\quad 45$

Intersectoral Action $\quad 46$

Key Policy Proposals $\quad 46$

$\begin{array}{ll}\text { 1. Health } & 47\end{array}$

2. Participation 51

3. Security

WHO and Ageing $\quad 54$

International Collaboration $\quad 55$

$\begin{array}{ll}\text { Conclusion } & 55\end{array}$

$\begin{array}{ll}\text { 6. References } & 57\end{array}$ 


\section{How Old is Older?}

This booklet uses the United Nations standard of age 60 to describe "older" people. This may seem young in the developed world and in those developing countries where major gains in life expectancy have already occurred. However, whatever age is used within different contexts, it is important to acknowledge that chronological age is not a precise marker for the changes that accompany ageing. There are dramatic variations in health status, participation and levels of independence among older people of the same age. Decision-makers need to take this into account when designing policies and programmes for their "older" populations. Enacting broad social policies based on chronological age alone can be discriminatory and counterproductive to well being in older age.

The hands you see in the background design of this paper are celebrating the worldwide triumph of population ageing. If you fan the pages quickly, you will see them applauding the important contribution that older people make to our societies, as well as the critical gains in public health and standards of living that have allowed people to live longer in almost all parts of the world.

This text and the preliminary version of the paper were drafted by Peggy Edwards, a Health Canada consultant based for six months at WHO, under the guidance of WHO's Ageing and Life Course Programme. The support from Health Canada at all phases of the project is gratefully acknowledged. 


\section{Introduction}

Population ageing raises many fundamental questions for policy-makers. How do we help people remain independent and active as they age? How can we strengthen health promotion and prevention policies, especially those directed to older people? As people are living longer, how can the quality of life in old age be improved? Will large numbers of older people bankrupt our health care and social security systems? How do we best balance the role of the family and the state when it comes to caring for people who need assistance, as they grow older? How do we acknowledge and support the major role that people play as they age in caring for others?

This paper is designed to address these questions and other concerns about population ageing. It targets government decision-makers at all levels, the nongovemmental sector and the private sector, all of whom are responsible for the formulation of policies and programmes on ageing. It approaches health from a broad perspective and acknowledges the fact that health can only be created and sustained through the participation of multiple sectors. It suggests that health providers and professionals must take a lead if we are to achieve the goal that healthy older persons remain a resource to their families, communities and economies, as stated in the WHO Brasilia Declaration on Ageing and Health in 1996.

- Part 1 describes the rapid worldwide growth of the population over age 60, especially in developing countries.

- Part 2 explores the concept and rationale for "active ageing" as a goal for policy and programme formulation.

- Part 3 summarizes the evidence about the factors that determine whether or not individuals and populations will enjoy a positive quality of life as they age.

- Part 4 discusses seven key challenges associated with an ageing population for governments, the nongovernmental, academic and private sectors.

- Part 5 provides a policy framework for active ageing and concrete suggestions for key policy proposals. These are intended to serve as a baseline for the development of more specific action steps at regional, national and local levels in keeping with the action plan adopted by the 2002 Second United Nations World Assembly on Ageing. 


\section{Global Ageing: A Triumph and a Challenge}

Population ageing is first and foremost a success story for public health policies as well as social and economic development. ...

Gro Harlem Brundtland, Director-General, World Health Organization, 1999

Population ageing is one of humanity's greatest triumphs. It is also one of our greatest challenges. As we enter the 21st century, global ageing will put increased economic and social demands on all countries. At the same time, older people are a precious, oftenignored resource that makes an important contribution to the fabric of our societies.

The World Health Organization argues that countries can afford to get old if governments, international organizations and civil society enact "active ageing" policies and programmes that enhance the health, participation and security of older citizens. The time to plan and to act is now.

\section{In all countries, and in developing countries in particular, measures to help older people remain healthy and active are a necessity, not a luxury.}

These policies and programmes should be based on the rights, needs, preferences and capacities of older people. They also need to embrace a life course perspective that recognizes the important influence of earlier life experiences on the way individuals age.

\section{The Demographic Revolution}

Worldwide, the proportion of people age 60 and over is growing faster than any other age group. Between 1970 and 2025, a growth in older persons of some 694 million or 223 percent is expected. In 2025, there will be a total of about 1.2 billion people over the age of 60 . By 2050 there will be 2 billion with 80 percent of them living in developing countries.

Age composition - that is, the proportionate numbers of children, young adults, middleaged adults and older adults in any given country - is an important element for policymakers to take into account. Population ageing refers to a decline in the proportion of children and young people and an increase in the proportion of people age 60 and over. As populations age, the triangular population pyramid of 2002 will be replaced with a more cylinder-like structure in 2025 (see Figure 1). 
Decreasing fertility rates and increasing longevity will ensure the continued "greying" of the world's population, despite setbacks in life expectancy in some African countries (due to AIDS) and in some newly independent states (due to increased deaths caused by cardiovascular disease and violence). Sharp decreases in fertility rates are being observed throughout the world. It is estimated that by 2025, 120 countries will have reached total fertility rates below replacement level (average fertility rate of 2.1 children per woman), a substantial increase compared to 1975, when just 22 countries had a total fertility rate below or equal to the replacement level. The current figure is 70 countries.
Until now, population ageing has been mostly associated with the more developed regions of the world. For example, currently nine of the ten countries with more than ten million inhabitants and the largest proportion of older people are in Europe (see Table 1). Little change in the ranking is expected by 2025 when people age 60 and over will make up about one-third of the population in countries like Japan, Germany and Italy, closely followed by other European countries (see Table 1).

\section{Figure 1. Global population pyramid in 2002 and 2025}

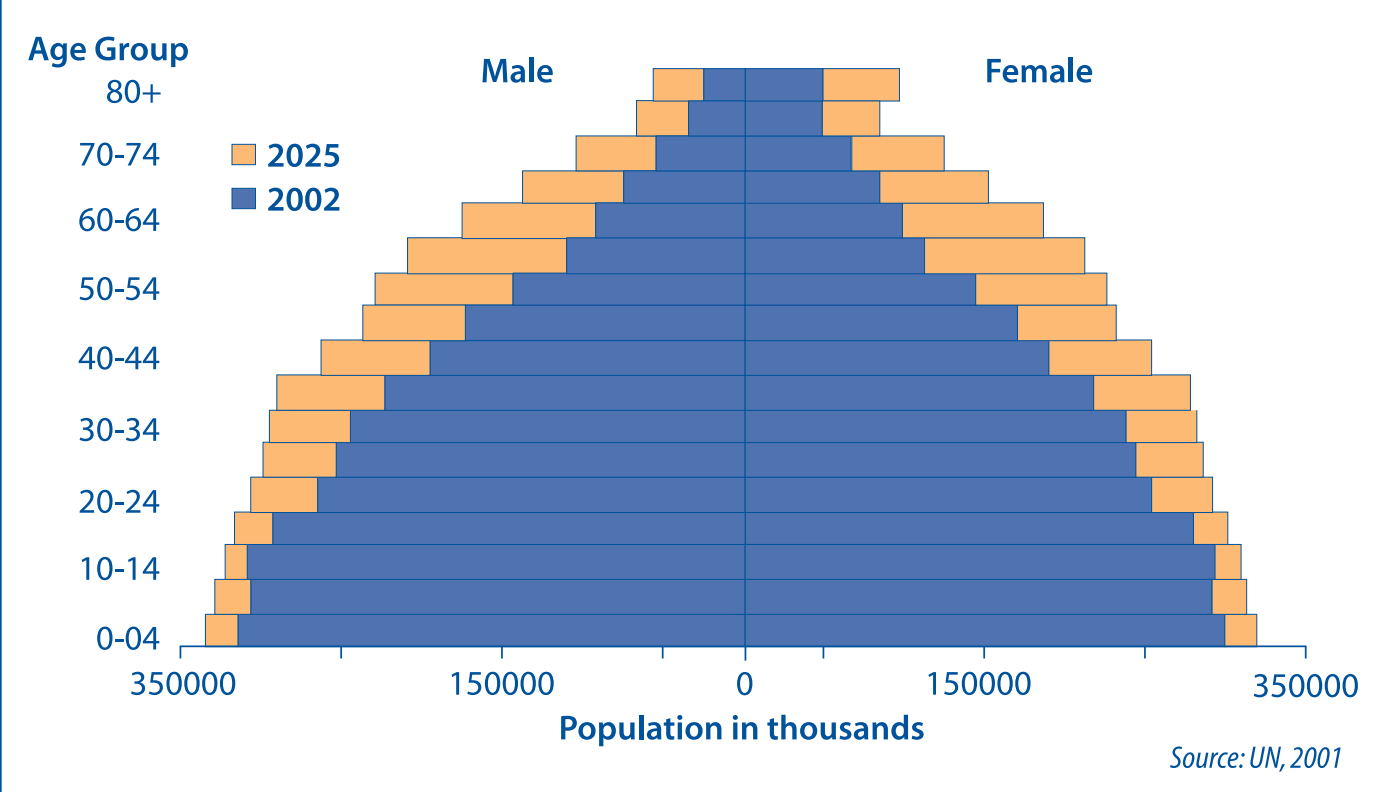

As the proportion of children and young people declines and the proportion of people age 60 and over increases, the triangular population pyramid of 2002 will be replaced with a more cylinder-like structure in 2025. 
Table 1. Countries with more than 10 million inhabitants (in 2002) with the highest proportion of persons above age 60

\begin{tabular}{|l|l|l|l|}
\hline \multicolumn{2}{|c|}{2002} & \multicolumn{2}{c|}{2025} \\
\hline Italy & $24.5 \%$ & Japan & $35.1 \%$ \\
\hline Japan & $24.3 \%$ & Italy & $34.0 \%$ \\
\hline Germany & $24.0 \%$ & Germany & $33.2 \%$ \\
\hline Greece & $23.9 \%$ & Greece & $31.6 \%$ \\
\hline Belgium & $22.3 \%$ & Spain & $31.4 \%$ \\
\hline Spain & $22.1 \%$ & Belgium & $31.2 \%$ \\
\hline Portugal & $21.1 \%$ & United Kingdom & $29.4 \%$ \\
\hline United Kingdom & $20.8 \%$ & Netherlands & $29.4 \%$ \\
\hline Ukraine & $20.7 \%$ & France & $28.7 \%$ \\
\hline France & $20.5 \%$ & Canada & $27.9 \%$ \\
\hline
\end{tabular}

Source:UN,2001

What is less known is the speed and significance of population ageing in less developed regions. Already, most older people - around 70 percent - live in developing countries (see Table 2). These numbers will continue to rise at a rapid pace.
In all countries, especially in developed ones, the older population itself is also ageing. People over the age of 80 currently number some 69 million, the majority of whom live in more developed regions. Although people over the age of 80 make up about one percent

Table 2.Absolute numbers of persons (in millions) above 60 years of age in countries with a total population approaching or above 100 million inhabitants (in 2002)

\begin{tabular}{|l|r|l|r|}
\hline \multicolumn{2}{|c|}{2002} & \multicolumn{2}{c|}{2025} \\
\hline China & 134.2 & China & 287.5 \\
\hline India & 81.0 & India & 168.5 \\
\hline United States of America & 46.9 & United States of America & 86.1 \\
\hline Japan & 31.0 & Japan & 43.5 \\
\hline Russian Federation & 26.2 & Indonesia & 35.0 \\
\hline Indonesia & 17.1 & Brazil & 33.4 \\
\hline Brazil & 14.1 & Russian Federation & 32.7 \\
\hline Pakistan & 8.6 & Pakistan & 18.3 \\
\hline Mexico & 7.3 & Bangladesh & 17.7 \\
\hline Bangladesh & 7.2 & Mexico & 17.6 \\
\hline Nigeria & 5.7 & Nigeria & 11.4 \\
\hline
\end{tabular}

Source:UN,2001 
of the world's population and three percent of the population in developed regions, this age group is the fastest growing segment of the older population.

In both developed and developing countries, the ageing of the population raises concerns about whether or not a shrinking labour force will be able to support that part of the population who are commonly believed to be dependent on others (i.e., children and older people).

The old-age dependency ratio (i.e., the total population age 60 and over divided by the population age 15 to 60 - see Table 3) is primarily used by economists and actuaries who forecast the financial implications of pension policies. However, it is also useful for those concerned with the management and planning of caring services.

\section{Old-age dependency ratios are changing quickly throughout the world. In Japan for ex ample, there are currently 39 people over age 60 for every 100 in the age group 15 - 60. In 2025 this number will increase to 66 .}

However, most of the older people in all countries continue to be a vital resource to their families and communities. Many continue to work in both the formal and informal labour sectors. Thus, as an indicator for forecasting population needs, the dependency ratio is of limited use. More sophisticated indices are needed to more accurately reflect "dependency", rather than falsely categorizing individuals that continue to be fully able and independent.

At the same time, active ageing policies and programmes are needed to enable people to continue to work according to their capacities and preferences as they grow older, and to prevent or delay disabilities and chronic diseases that are costly to individuals, families and the health care system. This is discussed further in the section on work (page 31) and in Challenge 2: Increased Risk of Disability (page 34) and Challenge 6: the Economics of an Ageing Population (page 42).

Table 3. Old age dependency ratio for selected countries / regions

\begin{tabular}{|l|c|l|l|}
\hline \multicolumn{2}{|c|}{2002} & \multicolumn{2}{c|}{2025} \\
\hline Japan & 0.39 & Japan & 0.66 \\
\hline North America & 0.26 & North America & 0.44 \\
\hline $\begin{array}{l}\text { European } \\
\text { Union }\end{array}$ & 0.36 & $\begin{array}{l}\text { European } \\
\text { Union }\end{array}$ & 0.56 \\
\hline
\end{tabular}

Source:UN, 2001

\section{Rapid Population Ageing in Developing Countries}

In 2002, almost 400 million people aged 60 and over lived in the developing world. By 2025, this will have increased to approximately 840 million representing 70 percent of all older people worldwide. (see Figure 2). In terms of regions, over half of the world's older people live in Asia. Asia's share of the world's oldest people will continue to increase the most while Europe's share as a proportion of the global older population will decrease the most over the next two decades (see Figure 3). 


\section{Figure 2. The numbers of people over age 60 in less and more developed regions,}

1970,2000 and 2025

\section{millions}

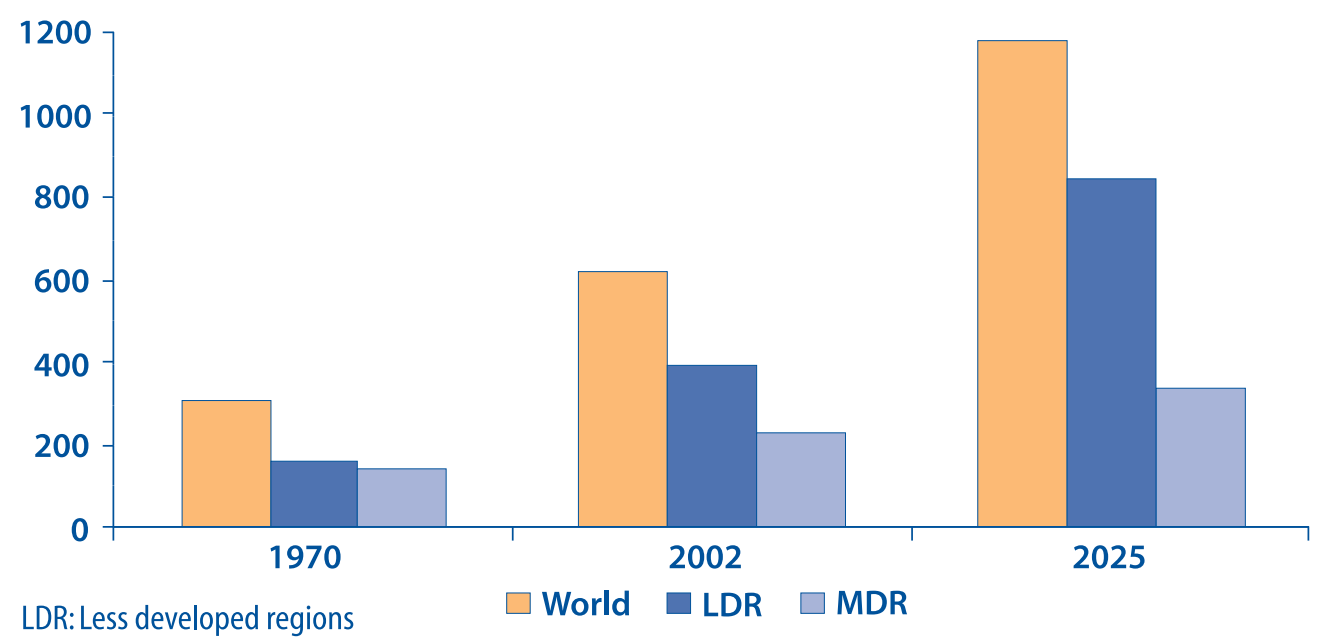

MDR: More developed regions

Figure 3. Distribution of world population over age 60 by region, 2002 and 2025
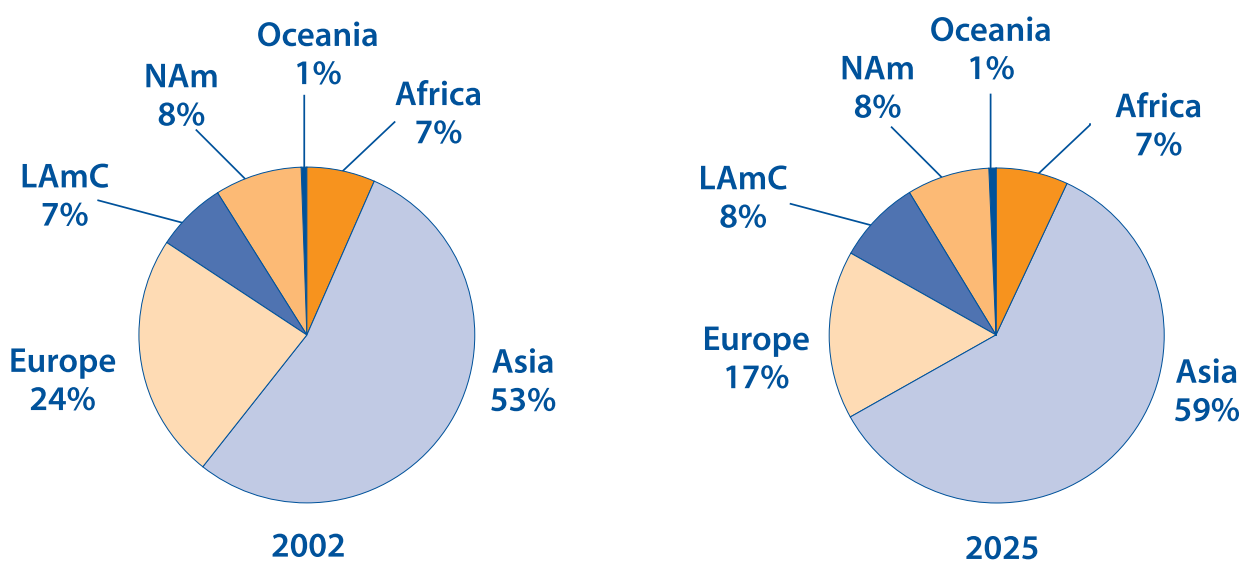

NAm: North America

LAmC: Latin America and the Caribbean 
Compared to the developed world, socioeconomic development in developing countries has often not kept pace with the rapid speed of population ageing. For example, while it took 115 years for the proportion of older people in France to double from 7 to 14 percent, it will take China only 27 years to achieve the same increase. In most of the developed world, population ageing was a gradual process following steady socio-economic growth over several decades and generations. In developing countries, the process is being compressed into two or three decades. Thus, while developed countries grew affluent before they became old, developing countries are getting old before a substantial increase in wealth occurs (Kalache and Keller, 2000).

Rapid ageing in developing countries is accompanied by dramatic changes in family structures and roles, as well as in labour patterns and migration. Urbanization, the migration of young people to cities in search of jobs, smaller families and more women entering the formal workforce mean that fewer people are available to care for older people when they need assistance. 


\section{Active Ageing: The Concept and Rationale}

If ageing is to be a positive experience, longer life must be accompanied by continuing opportunities for health, participation and security. The World Health Organization has adopted the term "active ageing" to express the process for achieving this vision.

\section{What is "Active Ageing"?}

\section{Active ageing is the process of optimizing opportunities for health, participation and security in order to enhance quality of life as people age.}

Active ageing applies to both individuals and population groups. It allows people to realize their potential for physical, social, and mental well being throughout the life course and to participate in society according to their needs, desires and capacities, while providing them with adequate protection, security and care when they require assistance.

The word "active" refers to continuing participation in social, economic, cultural, spiritual and civic affairs, not just the ability to be physically active or to participate in the labour force. Older people who retire from work and those who are ill or live with disabilities can remain active contributors to their families, peers, communities and nations. Active ageing aims to extend healthy life expectancy and quality of life for all people as they age, including those who are frail, disabled and in need of care.

"Health" refers to physical, mental and social well being as expressed in the WHO definition of health. Thus, in an active ageing framework, policies and programmes that promote mental health and social connections are as important as those that improve physical health status.

Maintaining autonomy and independence as one grows older is a key goal for both individuals and policy makers (see box on definitions). Moreover, ageing takes place within the context of others - friends, work associates, neighbours and family members. This is why interdependence as well as intergenerational solidarity (two-way giving and receiving between individuals as well as older and younger generations) are important tenets of active ageing. Yesterday's child is today's adult and tomorrow's grandmother or grandfather. The quality of life they will enjoy as grandparents depends on the risks and opportunities they experienced throughout the life course, as well as the manner in which succeeding generations provide mutual aid and support when needed. 


\section{Some key definitions}

Autonomy is the perceived ability to control,cope with and make personal decisions about how one lives on a day-to-day basis, according to one's own rules and preferences.

Independence is commonly understood as the ability to perform functions related to daily living - i.e. the capacity of living independently in the community with no and/or little help from others.

Quality of life is "an individual's perception of his or her position in life in the context of the culture and value system where they live, and in relation to their goals, expectations, standards and concerns. It is a broad ranging concept, incorporating in a complex way a person's physical health, psychological state, level of independence, social relationships, personal beliefs and relationship to salient features in the environment." (WHO, 1994). As people age, their quality of life is largely determined by their ability to maintain autonomy and independence.

Healthy life expectancy is commonly used as a synonym for "disability-free life expectancy".While life expectancy at birth remains an important measure of population ageing, how long people can expect to live without disabilities is especially important to an ageing population.

With the exception of autonomy which is notoriously difficult to measure, all of the above concepts have been elaborated by attempts to measure the degree of difficulty an older person has in performing activities related to daily living (ADLs) and instrumental activities of daily living (IADLS). ADLs include, for example, bathing, eating, using the toilet and walking across the room. IADLs include activities such as shopping, housework and meal preparation. Recently, a number of validated, more holistic measures of health-related quality of life have been developed. These indices need to be shared and adapted for use in a variety of cultures and settings.

The term "active ageing" was adopted by the World Health Organization in the late 1990s. It is meant to convey a more inclusive message than "healthy ageing" and to recognize the factors in addition to health care that affect how individuals and populations age (Kalache and Kickbusch, 1997).

The active ageing approach is based on the recognition of the human rights of older people and the United Nations Principles of independence, participation, dignity, care and self-fulfillment. It shifts strategic planning away from a "needs-based" approach (which assumes that older people are passive targets) to a "rights- based" approach that recognizes the rights of people to equality of opportunity and treatment in all aspects of life as they grow older. It supports their responsibility to exercise their participation in the political process and other aspects of community life. 


\section{A Life Course Approach to Active Ageing}

A life course perspective on ageing recognizes that older people are not one homogeneous group and that individual diversity tends to increase with age. Interventions that create supportive environments and foster healthy choices are important at all stages of life (see Figure 4).

As individuals age, noncommunicable diseases (NCDs) become the leading causes of morbidity, disability and mortality in all regions of the world, including in developing countries, as shown in Figures 5 and 6. NCDs, which are essentially diseases of later life, are costly to individuals, families and the public purse. But many NCDs are preventable or can be postponed. Failing to prevent or manage the growth of NCDs appropriately will result in enormous human and social costs that will absorb a disproportionate amount of resources, which could have been used to address the health problems of other age groups.

\section{Figure 4. Maintaining functional capacity over the life course}

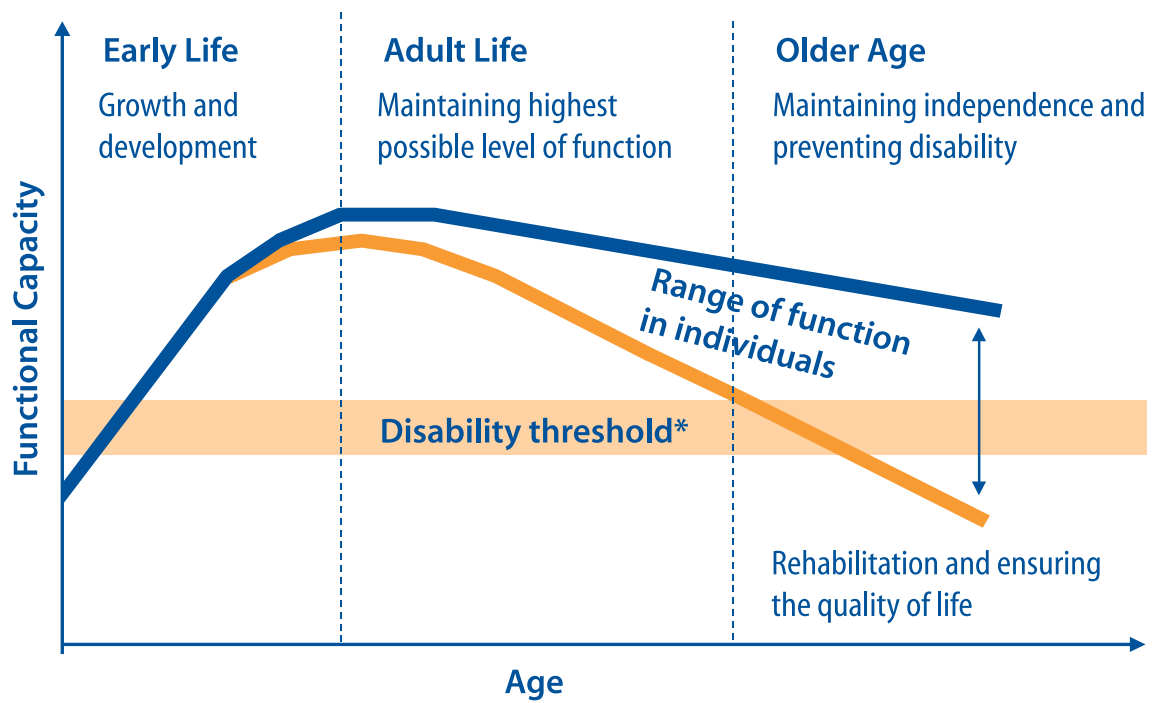

Source: Kalache and Kickbusch, 1997

*Changes in the environment can lower the disability threshold, thus decreasing the number of disabled peoplein a given community.

Functional capacity (such as ventilatory capacity, muscular strength, and cardiovascular output) increases in childhood and peaks in early adulthood, eventually followed by a decline. The rate of decline, however, is largely determined by factors related to adult lifestyle - such as smoking, alcohol consumption, levels of physical activity and diet - as well as external and environmental factors. The gradient of decline may becomeso steep as to result in premature disability. However,theacceleration in decline can be influenced and may be reversibleat any age through individual and public policy measures. 


\section{Figure 5. Leading causes of death, both sexes, 1998, low- and middle-income countries by age}

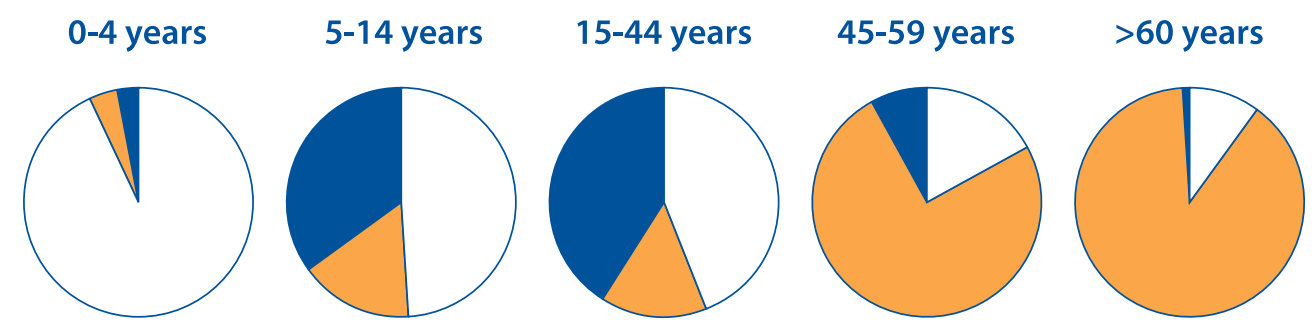

\footnotetext{
Noncommunicable conditions

Injuries

Communicable diseases, maternal and perinatal conditions and nutritional deficiencies
}

\section{Figure 6. Leading causes of burden of disease, both sexes, 1998,}

low- and middle-income countries by age

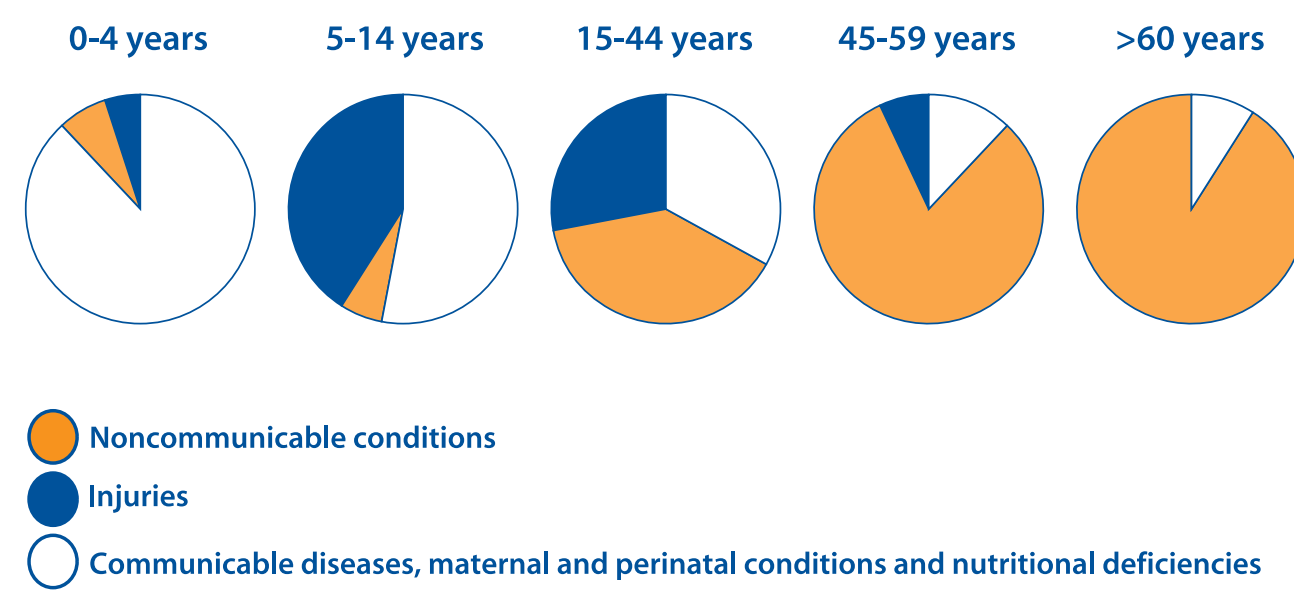


Major chronic conditions affecting

older people worldwide

- Cardiovascular diseases (such as coronary heart disease)

- Hypertension

- Stroke

- Diabetes

- Cancer

- Chronic obstructive pulmonary disease

- Musculoskeletal conditions (such as arthritis and osteoporosis)

- Mental health conditions (mostly dementia and depression)

- Blindness and visual impairment

Note:The causes of disability in older age are similar for women and men although women are more likely to report musculoskeletal problems.

Source:WHO, 1998a

In the early years, communicable diseases, maternal and perinatal conditions and nutritional deficiencies are the major causes of death and disease. In later childhood, adolescence and young adulthood, injuries and noncommunicable conditions begin to assume a much greater role. By midlife (age 45) and in the later years, NCDs are responsible for the vast majority of deaths and diseases (see Figures 5 and 6 ). Research is increasingly showing that the origins of risk for chronic conditions, such as diabetes and heart disease, begin in early childhood or even earlier. This risk is subsequently shaped and modified by factors, such as socio-economic status and experiences across the whole life span. The risk of developing NCDs continues to increase as individuals age. But it is tobacco use, lack of physical activity, inadequate diet and other established adult risk factors which will put individuals at relatively greater risk of developing NCDs at older ages (see Figure 7). Thus, it is important to address the risks of noncommunicable disease from early life to late life, i.e. throughout the life course.

\section{Active Ageing Policies and Programmes}

An active ageing approach to policy and programme development has the potential to address many of the challenges of both individual and population ageing. When health, labour market, employment, education and social policies support active ageing there will potentially be:

- fewer premature deaths in the highly productive stages of life

- fewer disabilities associated with chronic diseases in older age

- more people enjoying a positive quality of life as they grow older

- more people participating actively as they age in the social, cultural, economic and political aspects of society, in paid and unpaid roles and in domestic, family and community life

- lower costs related to medical treatment and care services. 
Active ageing policies and programmes recognize the need to encourage and balance personal responsibility (self-care), age-friendly environments and intergenerational solidarity. Individuals and families need to plan and prepare for older age, and make personal efforts to adopt positive personal health practices at all stages of life. At the same time supportive environments are required to "make the healthy choices the easy choices."

There are good economic reasons for enacting policies and programmes that promote active ageing in terms of increased participation and reduced costs in care. People who remain healthy as they age face fewer impediments to continued work. The current trend toward early retirement in industrialised countries is largely the result of public policies that have encouraged early withdrawal from the labour force. As populations age, there will be increasing pressures for such policies to change - particularly if more and more individuals reach old age in good health, i.e. are "fit for work." This would help to offset the rising costs in pensions and income security schemes as well as those related to medical and social care costs.

With regard to rising public expenditures for medical care, available data increasingly indicate that old age itself is not associated with increased medical spending. Rather, it is disability and poor health - often associated with old age - that are costly. As people age in better health, medical spending may not increase as rapidly.

\section{Figure 7. Scope for noncommunicable diseases prevention, a life course approach}

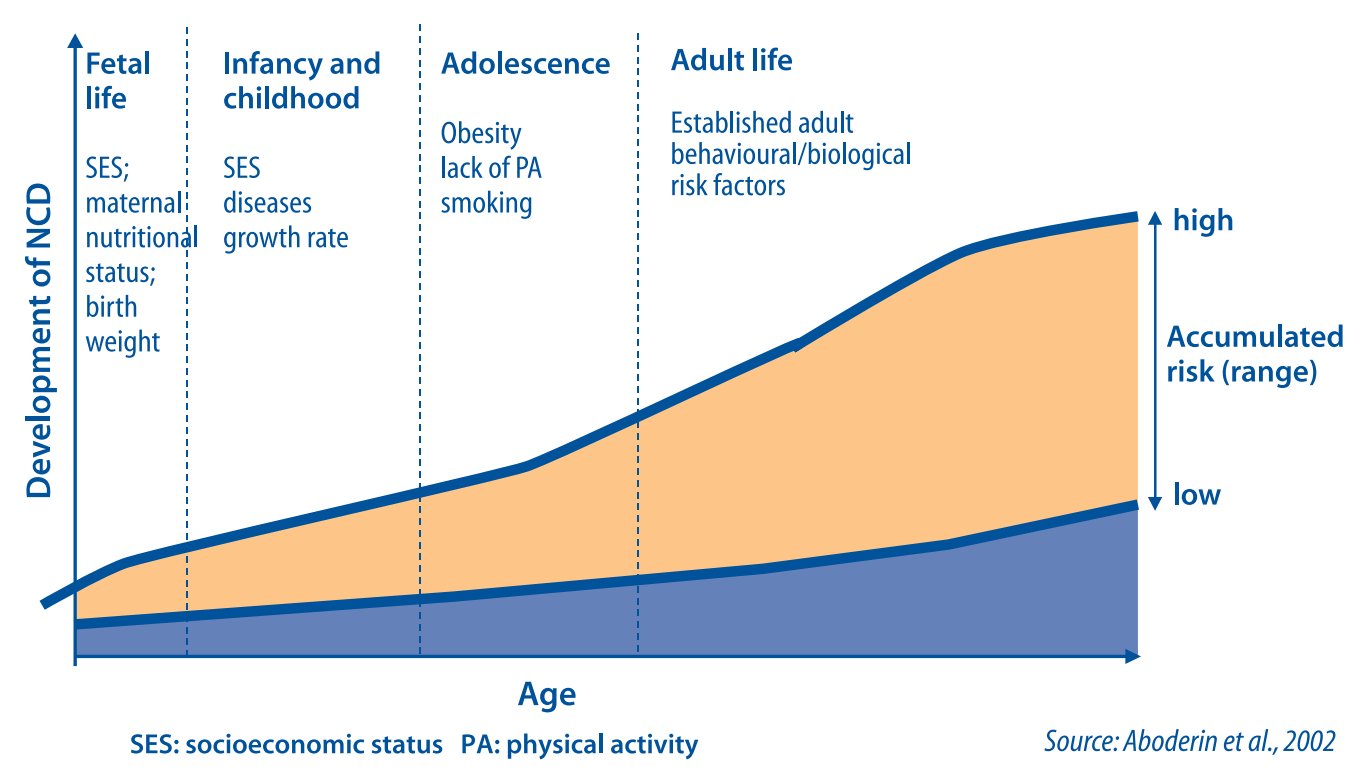


Policymakers need to look at the full picture and consider the savings achieved by declines in disability rates. In the USA for example, such declines might lower medical spending by about 20 percent over the next 50 years (Cutler, 2001). Between 1982 and 1994, in the USA, the savings in nursing home costs alone were estimated to exceed $\$ 17$ billion (Singer and Manton, 1998). Moreover, if increased numbers of healthy older people were to extend their participation in the work force (through either full or part-time employment), their contribution to public revenues would continuously increase. Finally, it is often less costly to prevent disease than to treat it. For example, it has been estimated that a one-dollar investment in measures to encourage moderate physical activity leads to a cost saving of $\$ 3.2$ in medical costs (U.S. Centers for Disease Control, 1999). 


\section{The Determinants of Active Ageing: Understanding the Evidence}

Active ageing depends on a variety of influences or "determinants" that surround individuals, families and nations. Understanding the evidence we have about these determinants helps us design policies and programmes that work.

The following section summarizes what we know about how the broad determinants of health affect the process of ageing. These determinants apply to the health of all age groups, although the emphasis here is on the health and quality of life of older persons. At this point, it is not possible to attribute direct causation to any one determinant; however, the substantial body of evidence on what determines health suggests that all of these factors (and the interplay between them) are good predictors of how well both individuals and populations age. More research is needed to clarify and specify the role of each determinant, as well as the interaction between determinants, in the active ageing process. We also need to better understand the pathways that explain how these broad determinants actually affect health and well being.

Moreover, it is helpful to consider the influence of various determinants over the life course so as to take advantage of transitions and "windows of opportunity" for enhancing health, participation and security at different stages. For example, there is evidence that stimulation and secure attachments in infancy influence an individual's ability to learn and

\section{Figure 8. The determinants of Active Ageing}

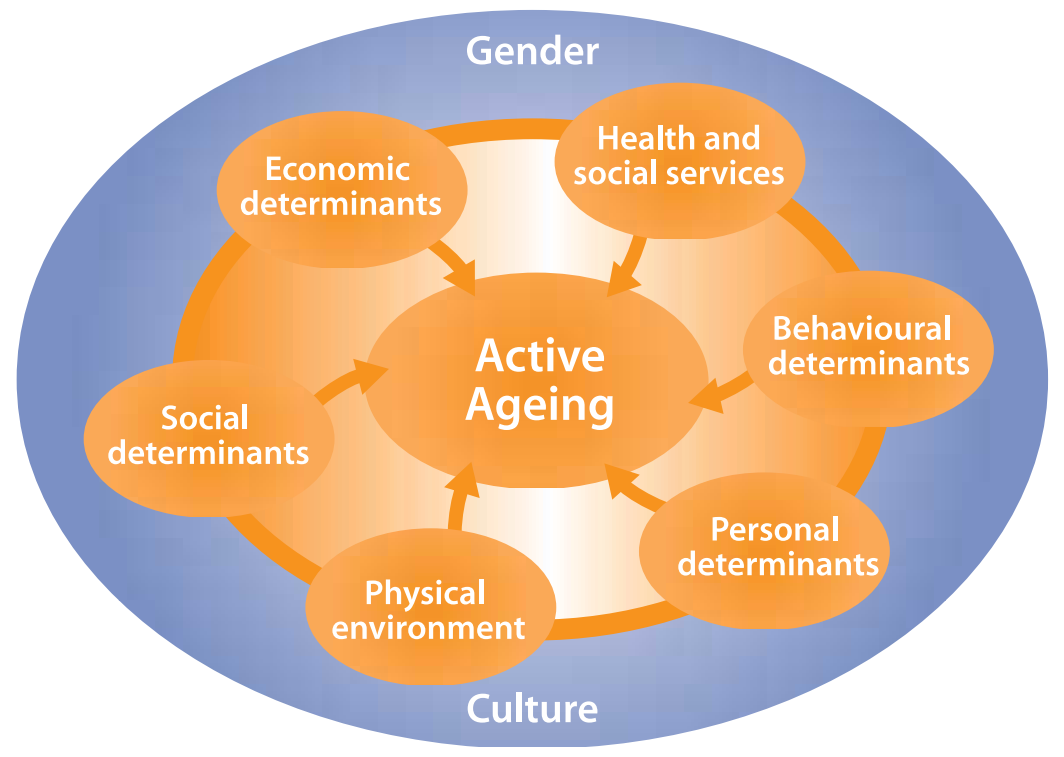


get along with others throughout all of the later stages of life. Employment, which is a determinant throughout adult life greatly influences one's financial readiness for old age. Access to high quality, dignified long-term care is particularly important in later life. Often, as is the case with exposure to pollution, the young and the old are the most vulnerable population groups.

\section{Cross-Cutting Determinants: Culture and Gender}

Culture is a cross-cutting determinant within the framework for understanding active ageing.

\section{Culture, which surrounds all indi- viduals and populations, shapes the way in which we age because it influ- ences all of the other determinants of active ageing.}

Cultural values and traditions determine to a large extent how a given society views older people and the ageing process. When societies are more likely to attribute symptoms of disease to the ageing process, they are less likely to provide prevention, early detection and appropriate treatment services. Culture is a key factor in whether or not co-residency with younger generations is the preferred way of living. For example, in most Asian countries, the cultural norm is to value extended families and to live together in multigenerational households. Cultural factors also influence health-seeking behaviours. For example, attitudes toward smoking are gradually changing in a range of countries.
There is enormous cultural diversity and complexity within countries and among countries and regions of the world. For example, diverse ethnicities bring a variety of values, attitudes and traditions to the mainstream culture within a country. Policies and programmes need to respect current cultures and traditions while de-bunking outdated stereotypes and misinformation. Moreover, there are critical universal values that transcend culture, such as ethics and human rights.

\section{Gender is a "lens" through which to consider the appropriateness of vari- ous policy options and how they will affect the well being of both men and women.}

In many societies, girls and women have lower social status and less access to nutritious foods, education, meaningful work and health services. Women's traditional role as family caregivers may also contribute to their increased poverty and ill health in older age. Some women are forced to give up paid employment to carry out their caregiving responsibilities. Others never have access to paid employment because they work full-time in unpaid caregiving roles, looking after children, older parents, spouses who are ill and grandchildren. At the same time, boys and men are more likely to suffer debilitating injuries or death due to violence, occupational hazards, and suicide. They also engage in more risktaking behaviours such as smoking, alcohol and drug consumption and unnecessary exposure to the risk of injury. 


\section{Determinants Related to Health and Social Service Systems}

\section{To promote active ageing, health systems need to take a life course perspective that focuses on health promotion, disease prevention and equitable access to quality primary health care and long-term care.}

Health and social services need to be integrated, coordinated and cost-effective. There must be no age discrimination in the provision of services and service providers need to treat people of all ages with dignity and respect.

\section{Health Promotion and Disease Prevention}

Health promotion is the process of enabling people to take control over and to improve their health. Disease prevention includes the prevention and management of the conditions that are particularly common as individuals age: noncommunicable diseases and injuries. Prevention refers both to "primary" prevention (e.g. avoidance of tobacco use) as well as "secondary" prevention (e.g. screening for the early detection of chronic diseases), or "tertiary" prevention, e.g. appropriate clinical management of diseases. All contribute to reducing the risk of disabilities. Disease prevention strategies - which may also address infectious diseases - save money at any age. For example, vaccinating older adults against influenza saves an estimated $\$ 30$ to $\$ 60$ in treatment costs per $\$ 1$ spent on vaccines (U.S. Department of Health and Human Services, 1999).

\section{Curative Services}

Despite best efforts in health promotion and disease prevention, people are at increasing risk of developing diseases as they age. Thus access to curative services becomes indispensable. As the vast majority of older persons in any given country live in the community, most curative services must be offered by the primary health care sector. This sector is best equipped to. make referrals to the secondary and tertiary levels of care where most acute and emergency care is also provided.

Ultimately, the worldwide shift in the global burden of disease toward chronic diseases requires a shift from a "find it and fix it" model to a coordinated and comprehensive continuum of care. This will require a reorientation in health systems that are currently organized around acute, episodic experiences of disease. The present acute care models of health service delivery are inadequate to address the health needs of rapidly ageing populations (WHO, 2001).

As the population ages, the demand will continue to rise for medications that are used to delay and treat chronic diseases, alleviate pain and improve quality of life. This calls for a renewed effort to increase affordable access to essential safe medications and to better ensure the appropriate, cost-effective use of current and new drugs. Partners in this effort need to include governments, health professionals, the pharmaceutical industry, traditional healers, employers and organizations representing older people. 


\section{Long-term care}

Long-term care is defined by WHO as "the system of activities undertaken by informal caregivers (family, friends and/or neighbours) and/or professionals (health and social services) to ensure that a person who is not fully capable of self-care can maintain the highest possible quality of life, according to his or her individual preferences, with the greatest possible degree of independence, autonomy, participation, personal fulfillment and human dignity" (WHO, 2000b).

Thus, long-term care includes both informal and formal support systems. The latter may include a broad range of community services (e.g., public health, primary care, home care, rehabilitation services and palliative care) as well as institutional care in nursing homes and hospices. It also refers to treatments that halt or reverse the course of disease and disability.

\section{Mental Health Services}

Mental health services, which play a crucial role in active ageing, should be an integral part of long-term care. Particular attention needs to be paid to the under-diagnosis of mental illness (especially depression) and to suicide rates among older people (WHO, 2001a).

\section{Behavioural Determinants}

$$
\begin{aligned}
& \text { The adoption of healthy lifestyles } \\
& \text { and actively participating in one's } \\
& \text { own care are important at all stages } \\
& \text { of the life course. One of the myths } \\
& \text { of ageing is that it is too late to adopt } \\
& \text { such lifestyles in the later years. On } \\
& \text { the contrary, engaging in appropri- } \\
& \text { ate physical activity, healthy eating, } \\
& \text { not smoking and using alcohol and } \\
& \text { medications wisely in older age can } \\
& \text { prevent disease and functional de- } \\
& \text { cline, ex tend longevity and enhance } \\
& \text { one's quality of life. }
\end{aligned}
$$

\section{Tobacco Use}

Smoking is the most important modifiable risk factor for NCDs for young and old alike and a major preventable cause of premature death. Smoking not only increases the risk for diseases such as lung cancer, it is also negatively related to factors that may lead to important losses in functional capacity. For example, smoking accelerates the rate of decline of bone density, muscular strength and respiratory function. Research on the effects of smoking revealed not just that smoking is a risk factor for a large and increasing number of diseases but also that its ill effects are cumulative and long lasting. The risk of contracting at least one of the diseases associated with smoking increases with the duration and the amount of exposure. 
A critical message for young people should always be "If you want to grow older, don't smoke. Moreover, if you want to grow older and to increase your chance to age well, again don't smoke."

The benefits of quitting are wide-ranging and apply to any age group. It is never too late to quit smoking. For instance, stroke risk decreases after two years of abstinence from cigarette smoking and, after five years, it becomes the same as that for individuals who have never smoked. For other diseases, e.g. lung cancer and obstructive pulmonary disease, quitting decreases the risk but only very slowly. Thus, current exposure is not a very good indicator of current and future risks and past exposure should be taken into account as well; the effects of smoking are cumulative and long standing (Doll, 1999).

\section{Smoking may interfere with the effect of} needed medications. Exposure to second-hand smoke can also have a negative effect on older people's health, especially if they suffer from asthma or other respiratory problems.

Most smokers start young and are quickly addicted to the nicotine in tobacco. Therefore, efforts to prevent children and youth from starting to smoke must be a primary strategy in to bacco control. At the same time, it is important to reduce the demand for tobacco among adults (through comprehensive actions such as taxation and restrictions on advertising) and to help adults of all ages to quit. Studies have shown that tobacco control is highly cost-effective in low- and middle-income countries. In China, for example, conservative estimates suggest that a 10 percent increase in tobacco taxes would reduce consumption by five percent and increase overall revenue by five percent. This increased revenue would be sufficient to finance a package of essential health care services for one-third of China's poorest citizens (World Bank, 1999).

\section{Physical Activity}

Participation in regular, moderate physical activity can delay functional declines. It can reduce the onset of chronic diseases in both healthy and chronically ill older people. For example, regular moderate physical activity reduces the risk of cardiac death by 20 to 25 percent among people with established heart disease (Merz and Forrester, 1997). It can also substantially reduce the severity of disabilities associated with heart disease and other chronic illnesses (U.S Preventive Services Task Force, 1996). Active living improves mental health and often promotes social contacts. Being active can help older people remain as independent as possible for the longest period of time. It can also reduce the risk of falls. There are thus important economic benefits when older people are physically active. Medical costs are substantially lower for older people who are active (WHO, 1998).

Despite all of these benefits, high proportions of older people in most countries lead sedentary lives. Populations with low incomes, ethnic minorities and older people with disabilities are the most likely to be inactive. Policies and programmes should encourage inactive people to become more active as they age and to provide them with opportunities to do so. It is particularly important to provide safe areas for walking and to support culturally-appropriate community activities that stimulate physical 
activity and are organized and led by older people themselves. Professional advice to "go from doing nothing to doing something" and physical rehabilitation programmes that help older people recover from mobility problems are both effective and cost-efficient.

In the least developed countries, the opposite problem may occur. In these countries, individuals are often engaged in strenuous physical work and chores that may hasten disabilities, cause injuries and aggravate previous conditions, especially as they approach old age. This may include heavy caregiving responsibilities for ill and dying relatives. Health promotion efforts in these areas should be directed at providing relief from repetitive, strenuous tasks and making adjustments to unsafe physical movements at work that will decrease injuries and pain. Older people who regularly engage in vigorous physical work need opportunities for rest and recreation.

\section{Healthy Eating}

Eating and food security problems at all ages include both under-nutrition (mostly, but not exclusively, in the least developed countries) and excess energy intake. In older people, malnutrition can be caused by limited access to food, socioeconomic hardships, a lack of information and knowledge about nutrition, poor food choices (e.g., eating high fat foods), disease and the use of medications, tooth loss, social isolation, cognitive or physical disabilities that inhibit one's ability to buy foods and prepare them, emergency situations and a lack of physical activity.

Excess energy intake greatly increases the risk for obesity, chronic diseases and disabilities as people grow older.

\section{Diets high in (saturated) fat and salt, low in fruits and vegetables and providing insufficient amounts of fibre and vitamins combined with sedentarism, are major risks factors for chronic conditions like diabetes, cardiovascular disease, high blood pressure, obesity, arthritis and some cancers.}

Insufficient calcium and vitamin D is associated with a loss of bone density in older age and consequently an increase in painful, costly and debilitating bone fractures, especially in older women. In populations with high fracture incidence, risk can be decreased through ensuring adequate calcium and vitamin D intake.

\section{Oral Health}

Poor oral health - primarily dental caries, periodontal diseases, tooth loss and oral cancer - cause other systemic health problems. They create a financial burden for individuals and society and can reduce self-confidence and quality of life. Studies show that poor oral health is associated with malnutrition and therefore increased risks for various noncommunicable diseases. Oral health promotion and cavity prevention programmes designed to encourage people to keep their natural teeth need to begin early in life and continue over the life course. Because of the pain and reduced quality of life associated with oral health problems, basic dental treatment services and accessibility to dentures are required. 


\section{Alcohol}

While older people tend to drink less than younger people, metabolism changes that accompany ageing increase their susceptibility to alcohol-related diseases, including malnutrition and liver, gastric and pancreatic diseases. Older people also have greater risks for alcohol-related falls and injuries, as well as the potential hazards associated with mixing alcohol and medications. Treatment services for alcohol problems should be available to older people as well as younger people.

According to a recent WHO review of the literature, there is evidence that alcohol use at very low levels (up to one drink a day) may offer some form of protection against coronary heart disease and stroke for people age 45 and over. However, in terms of overall excess mortality, the adverse effects of drinking outweigh any protection against coronary heart disease, even in high risk populations (Jemigan et al., 2000).

\section{Medications}

Because older people often have chronic health problems, they are more likely than younger people to need and use medications - traditional, over-the-counter and prescribed. In most countries, older people with low incomes have little or no access to insurance for medications. As a result, many go without or spend an inappropriately large part of their meager incomes on drugs.

In contrast, medications are sometimes overprescribed to older people (especially to older women) who have insurance or the means to pay for these drugs. Adverse drug-related reactions and falls associated with medication use (especially sleeping pills and tranquilizers) are significant causes of personal suffering and costly preventable hospital admissions (Gurwitz and Avorn, 1991).

Iatrogenesis - health problems that are induced by diagnoses or treatments - caused by the use of drugs is common in old age, due to the interaction of drugs, inadequate dosages and a higher frequency of unpredictable reactions through unknown mechanisms. With the advent of many new therapies, there is an increasing need to establish systems for preventing adverse drug reactions and for informing both health professionals and the ageing public about the risks and benefits of modern therapies.

\section{Adherence}

Access to needed medications is insufficient in itself unless adherence to long-term therapy for ageing-related chronic illnesses is high. Adherence includes the adoption and maintenance of a wide range of behaviours (e.g., healthy diet, physical activity, not smoking), as well as taking medications as directed by a health professional. It is estimated that in developed countries adherence to long-term therapy averages only 50 percent. In developing countries the rates are even lower. Such poor adherence severely compromises the effectiveness of treatments and has dramatic quality of life and economic implications for public health. Population health outcomes predicted by treatment efficacy data can only be achieved if adherence information is provided to all health professionals and planners. Without a system that addresses the influences on adherence, advances in biomedical technol- 
ogy will fail to realize their potential to reduce the burden of chronic disease (Dipollina and Sabate, 2002).

\section{Determinants Related to Personal Factors}

\section{Biology and Genetics}

Biology and genetics greatly influence how a person ages. Ageing is a set of biological processes that are genetically determined. Ageing can be defined as a progressive, generalized impairment of function resulting in a loss of adaptative response to a stress and in a growing risk of age-associated disease (Kirkwood, 1996). In other words, the main reason why older persons get sick more frequently than younger persons is that, due to their longer lives, they have been exposed to external, behavioural, and environmental factors that cause disease for a longer time than their younger counterparts (Gray, 1996).

\section{While genes may be involved in the causation of disease, for many diseases the cause is environmental and ex ternal to a greater degree than it is genetic and internal.}

It should also be noted that there is evidence in human populations that longevity tends to run in families. But, all things considered, there is general agreement that the lifelong trajectory of health and disease for an individual is the result of a combination of genetics, environment, lifestyle, nutrition, and to an important extent, chance (Kirkwood, 1996).
Therefore, the influence of genetics on the development of chronic conditions such as diabetes, heart disease, Alzheimer's Disease and certain cancers varies greatly among individuals. For many people, lifestyle behaviours such as not smoking, personal coping skills and a network of close kin and friends can effectively modify the influence of heredity on functional decline and the onset of disease.

\section{Psychological Factors}

Psychological factors including intelligence and cognitive capacity (for example, the ability to solve problems and adapt to change and loss) are strong predictors of active ageing and longevity (Smits et al., 1999). During normal ageing, some cognitive capacities (including learning speed and memory) naturally decline with age. However, these losses can be compensated by gains in wisdom, knowledge and experience. Often, declines in cognitive functioning are triggered by disuse (lack of practice), illness (such as depression), behavioural factors (such as the use of alcohol and medications), psychological factors (such as lack of motivation, low expectations and lack of confidence), and social factors (such as loneliness and isolation), rather than ageing per se.

Other psychological factors that are acquired across the life course greatly influence the way in which people age. Self-efficacy (the belief people have in their capacity to exert control over their lives) is linked to personal behaviour choices as one ages and to preparation for retirement. Coping styles determine how well people adapt to the transitions (such as retirement) and crises of ageing (such as bereavement and the onset of illness). 
Men and women who prepare for old age and are adaptable to change make a better adjustment to life after age 60. Most people remain resilient as they age and, on the whole, older people do not vary significantly from younger people in their ability to cope.

\section{Determinants Related to the Physical Environment}

\section{Physical Environments}

Physical environments that are age friendly can make the difference between independence and dependence for all individuals but are of particular importance for those growing older. For example, older people who live in an unsafe environment or areas with multiple physical barriers are less likely to get out and therefore more prone to isolation, depression, reduced fitness and increased mobility problems.

Specific attention must be given to older people who live in rural areas (some 60 percent worldwide) where disease patterns may be different due to environmental conditions and a lack of available support services. Urbanization and the migration of younger people in search of jobs may leave older people isolated in rural areas with little means of support and little or no access to health and social services.

Accessible and affordable public transportation services are needed in both rural and urban areas so that people of all ages can fully participate in family and community life. This is especially important for older persons who have mobility problems.

Hazards in the physical environment can lead to debilitating and painful injuries among older people. Injuries from falls, fires and traffic collisions are the most common.

\section{Safe Housing}

Safe, adequate housing and neighbourhoods are essential to the well being of young and old. For older people, location, including proximity to family members, services and transportation can mean the difference between positive social interaction and isolation. Building codes need to take the health and safety needs of older people into account. Household hazards that increase the risk of falling need to be remedied or removed.

Worldwide, there is an increasing trend for older people to live alone - especially unattached older women who are mainly widows and are often poor, even in developed countries. Others may be forced to live in arrangements that are not of their choice, such as with relatives in already crowded households. In many developing countries, the proportion of older people living in slums and shanty towns is rising quickly as many, who moved to the cities long ago, have become long-term slumdwellers, while other older people migrate to cities to join younger family members who have already moved there. Older people living in these settlements are at high risk for social isolation and poor health.

In times of crisis and conflict, displaced older people are particularly vulnerable. Often they are unable to walk to refugee camps. Even if they make it to camps, it may be hard to obtain shelter and food, especially for older women and older persons with disabilities who experience low social status and multiple other barriers. 


\section{Falls}

Falls among older people are a large and increasing cause of injury, treatment costs and death. Environmental hazards that increase the risks of falling include poor lighting, slippery or irregular walking surfaces and a lack of supportive handrails. Most often, these falls occur in the home environment and are preventable.

The consequences of injuries sustained in older age are more severe than among younger people. For injuries of the same severity, older people experience more disability, longer hospital stays, extended periods of rehabilitation, a higher risk of subsequent dependency and a higher risk of dying.

\section{The great majority of injuries are preventable; how ever, the tra ditional view of injuries as "accidents" has resulted in historical neglect of this area in public health.}

\section{Clean Water, Clean Air and Safe Foods}

Clean water, clean air and access to safe foods are particularly important for the most vulnerable population groups, i.e. children and older persons, and for those who have chronic illnesses and compromised immune systems.

\section{Determinants Related to the Social Environment}

Social support, opportunities for education and lifelong learning, peace, and protection from violence and abuse are key factors in the social environment that enhance health, participation and security as people age. Loneliness, social isolation, illiteracy and a lack of education, abuse and exposure to conflict situations greatly increase older people's risks for disabilities and early death.

\section{Social Support}

Inadequate social support is associated not only with an increase in mortality, morbidity and psychological distress but a decrease in overall general health and well being. Disruption of personal ties, loneliness and conflictual interactions are major sources of stress, while supportive social connections and intimate relations are vital sources of emotional strength (Gironda and Lubben, in press). In Japan, for example, older people who reported a lack of social contact were 1.5 times more likely to die in the next three years than were those with higher social support (Sugiswawa et al, 1994).

Older people are more likely to lose family members and friends and to be more vulnerable to loneliness, social isolation and the availability of a "smaller social pool". Social isolation and loneliness in old age are linked to a decline in both physical and mental well being. In most societies, men are less likely than women to have supportive social networks. However, in some cultures, older women who are widowed are systematically excluded from mainstream society or even rejected by their community.

Decision-makers, nongovernmental organizations, private industry and health and social service professionals can help foster social networks for ageing people by supporting traditional societies and community groups run by older people, voluntarism, neighbourhood helping, peer mentoring and visiting, family caregivers, intergenerational programmes and outreach services. 


\section{Violence and Abuse}

Older people who are frail or live alone may feel particularly vulnerable to crimes such as theft and assault. A common form of violence against older people (especially against older women) is "elder abuse" committed by family members and institutional caregivers who are well known to the victims. Elder abuse occurs in families at all economic levels. It is likely to escalate in societies experiencing economic upheaval and social disorganization when overall crime and exploitation tends to increase.

\section{According to the International Network for the Prevention of Elder Abuse, elder abuse is "a single or repeated act, or lack of appropriate action occurring within any rela- tionship where there is an ex pecta- tion of trust which causes harm or distress to an older person" (Action on Elder Abuse 1995).}

Elder abuse includes physical, sexual, psychological and financial abuse as well as neglect. Older people themselves perceive abuse as including the following societal factors: neglect (social exclusion and abandonment), violation (human, legal and medical rights) and deprivation (choices, decisions, status, finances and respect) (WHO/INPEA 2002). Elder abuse is a violation of human rights and a significant cause of injury, illness, lost productivity, isolation and despair. Typically, it is underreported in all cultures.
Confronting and reducing elder abuse requires a multisectoral, multidisciplinary approach involving justice officials, law enforcement officers, health and social service workers, labour leaders, spiritual leaders, faith institutions, advocacy organizations and older people themselves. Sustained efforts to increase public awareness of the problem and to shift values that perpetuate gender inequities and ageist attitudes are also required.

\section{Education and Literacy}

Low levels of education and illiteracy are associated with increased risks for disability and death among people as they age, as well as with higher rates of unemployment. Education in early life combined with opportunities for lifelong learning can help people develop the skills and confidence they need to adapt and stay independent, as they grow older.

Studies have shown that employment problems of older workers are often rooted in their relatively low literacy skills, not in ageing per se. If people are to remain engaged in meaningful and productive activities as they grow older, there is a need for continuous training in the workplace and lifelong learning opportunities in the community (OECD, 1998).

Like younger people, older citizens need training in new technologies, especially in agriculture and electronic communication. Self-directed learning, increased practice and physical adjustments (such as the use of large print) can compensate for reductions in visual acuity, hearing and short-term memory. Older people can and do remain creative and flexible. Intergenerational learning bridges age differences, enhances the transmission of cultural values and promotes the worth of all ages. Studies have shown that young people who learn with 
older people have more positive and realistic attitudes about the older generation.

Unfortunately, there continue to be striking disparities in literacy rates between men and women. In 1995 in the least developed countries, 31 percent of adult women were illiterate compared to 20 percent of adult men (WHO, 1998a).

\section{Economic Determinants}

Three aspects of the economic environment have a particularly significant effect on active ageing: income, work and social protection.

\section{Income}

Active ageing policies need to intersect with broader schemes to reduce poverty at all ages. While poor people of all ages face an increased risk of ill health and disabilities, older people are particularly vulnerable. Many older people especially those who are female, live alone or in rural areas do not have reliable or sufficient incomes. This seriously affects their access to nutritious foods, adequate housing and health care. In fact, studies have shown that older people with low incomes are onethird as likely to have high levels of functioning as those with high incomes (Guralnick and Kaplan, 1989).

The most vulnerable are older women and men who have no assets, little or no savings, no pensions or social security payments or who are part of families with low or uncertain incomes. Particularly, those without children or family members often face an uncertain future and are at high risk for homelessness and destitution.

\section{Social Protection}

In all countries of the world, families provide the majority of support for older people who require help. However, as societies develop and the tradition of generations living together begins to decline, countries are increasingly called on to develop mechanisms that provide social protection for older people who are unable to earn a living and are alone and vulnerable. In developing countries, older people who need assistance tend to rely on family support, informal service transfers and personal savings. Social insurance programmes in these settings are minimal and in some cases redistribute income to minorities in the population who are less in need. However, in countries such as South Africa and Namibia, which have a national old age pension, these benefits are a major source of income for many poor families as well as the older adults who live in these families. The money from these small pensions is used to purchase food for the household, to send children to school, to invest in farming technologies and to ensure survival for many urban poor families.

In developed countries, social security measures can include old-age pensions, occupational pension schemes, voluntary savings incentives, compulsory savings funds and insurance programmes for disability, sickness, long-term care and unemployment. In recent years, policy reforms have favoured a multi-pillared approach that mixes state and private support for old age security and encourages working longer and gradual retirement (OECD, 1998). 


\section{Work}

Throughout the world, if more people would enjoy opportunities for dignified work (properly remunerated, in adequate environments, protected against the hazards) earlier in life, people would reach old age able to participate in the workforce. Thus, the whole society would benefit. In all parts of the world, there is an increasing recognition of the need to support the active and productive contribution that older people can and do make in formal work, informal work, unpaid activities in the home and in voluntary occupations.

In developed countries, the potential gain of encouraging older people to work longer is not being fully realized. But when unemployment is high, there is often a tendency to see reducing the number of older workers as a way to create jobs for younger people. However, experience has shown that the use of early retirement to free up new jobs for the unemployed has not been an effective solution (OECD, 1998).

In less developed countries, older people are by necessity more likely to remain economically active into old age (see Figure 9). However, industrialization, adoption of new technologies and labour market mobility is threatening much of the traditional work of older people, particularly in rural areas. Development projects need to ensure that older people are eligible for credit schemes and full participation in incomegenerating opportunities.

\section{Figure 9. Percentage of labour force participation by people 65 and older, by region}

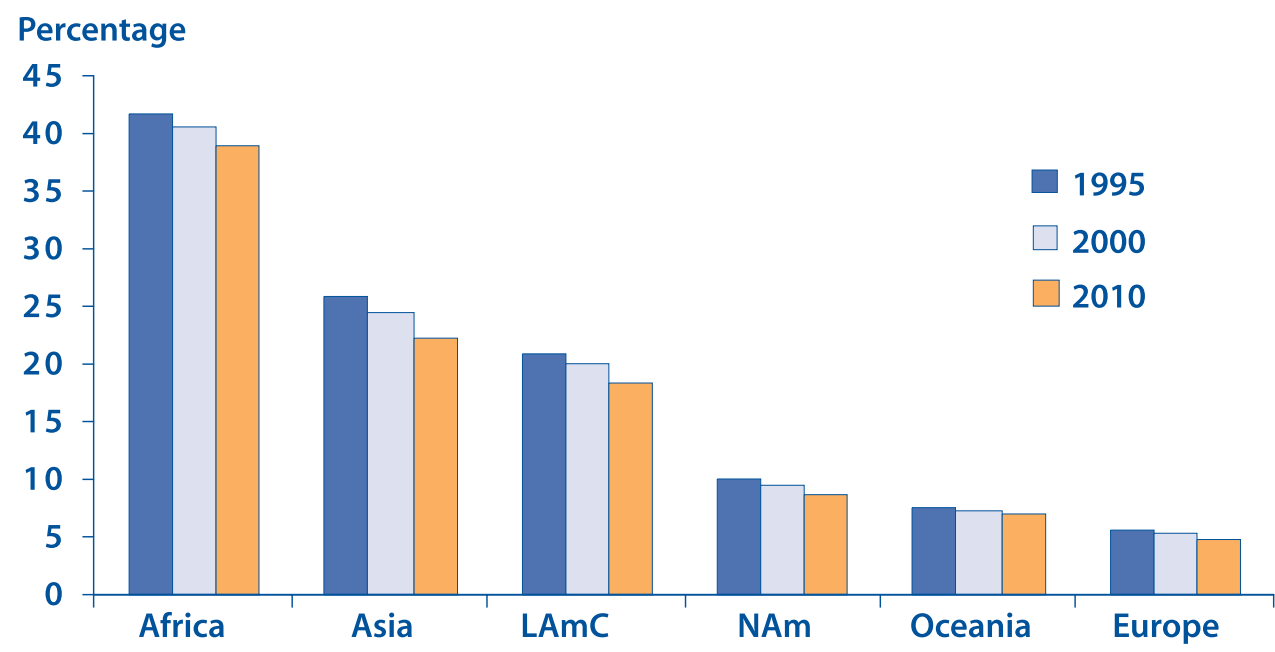

LAmC: Latin America and the Caribbean NAm: North America 
Concentrating only on work in the formal labour market tends to ignore the valuable contribution that older people make in work in the informal sector (e.g., small scale, self-employed activities and domestic work) and unpaid work in the home. childcare so that younger adults can work outside the home.

In all countries, skilled and experienced older people act as volunteers in schools, communities, religious institutions, businesses and health and political organizations. Voluntary work benefits older people by increasing social contacts and psychological well being while making a significant contribution to their communities and nations.

In both developing and developed countries, older people often take prime responsibility for household management and 


\section{Challenges of an Ageing Population}

The challenges of population ageing are global, national and local. Meeting these challenges will require innovative planning and substantive policy reforms in developed countries and in countries in transition. Developing countries, most of whom do not yet have comprehensive policies on ageing, face the biggest challenges.

\section{Challenge 1: The Double Burden of Disease}

As nations industrialize, changing patterns of living and working are inevitably accompanied by a shift in disease patterns. These changes impact developing countries most. Even as these countries continue to struggle with infectious diseases, malnutrition and complications from childbirth, they are faced with the rapid growth of noncommunicable diseases (NCDs). This "double burden of disease" strains already scarce resources to the limit.

The shift from communicable to NCDs is fast occurring in most of the developing world, where chronic illnesses such as heart disease, cancer and depression are quickly becoming the leading causes of morbidity and disability. This trend will escalate over the next few decades. In 1990, 51 percent of the global burden of disease in developing and newly industrialized countries was caused by NCDs, mental health disorders and injuries. By 2020, the burden of these diseases will rise to approximately 78 percent (See Figure 10).

\section{Figure 10. Global burden of disease 1990 and 2020 contribution by disease group} in developing and newly industrialized countries

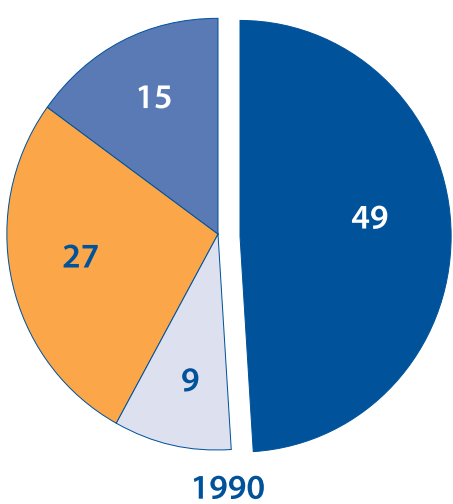

Communicable diseases Noncommunicable diseases

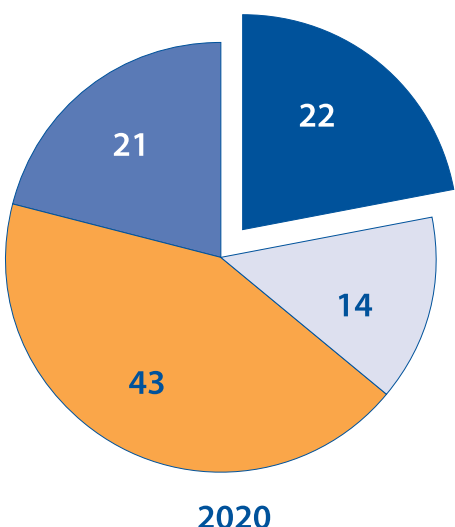

Neuropsychiatric diseases Injuries

Source:Murray\&Lopez, 1996

By 2020, over 70 percent of the global burden of disease in developing and newly industrialized countries will be caused by noncommunicable diseases, mental health disorders and injuries. 
There is no question that policy makers and donors must continue to put resources toward the control and eradication of infectious diseases. But it is also critical to put policies, programmes and intersectoral partnerships into place that can help to halt the massive expansion of chronic NCDs. While not necessarily easy to implement, those that focus on community development, health promotion, disease prevention and increasing participation are often the most effective in controlling the burden of disease. Furthermore other long-term policies that target malnutrition and poverty will help to reduce both chronic communicable and noncommunicable diseases.

Support for relevant research is most urgently needed for less developed countries. Currently, low and middle-income countries have 85 percent of the world's population and 92 percent of the disease burden, but only 10 percent of the world's health research spending (WHO, 2000).

\section{Challenge 2: Increased Risk of Disability}

In both developing and developed countries, chronic diseases are significant and costly causes of disability and reduced quality of life. An older person's independence is threatened when physical or mental disabilities make it difficult to carry out the activities of daily living.

As they grow older, people with disabilities are likely to encounter additional barriers related to the ageing process. For example, mobility problems due to poliomyelitis in childhood may be considerably aggravated later in life. Now that many young people with intellectual disabilities survive at much older ages and live beyond their parents, this special group also requires careful attention from policy makers.

Many people develop disabilities in later life related to the wear and tear of ageing (e.g., arthritis) or the onset of a chronic disease,

\section{HIV/AIDS and older people}

In Africa and other developing regions, HIV/AIDS has had multiple impacts on older people, in terms of living with the disease themselves, caring for others who are infected and taking on the parenting role with orphans of AIDS. This impact has been largely ignored to date. In fact, most data on HIV and AIDS infection rates are only compiled up to age 49. Improved data collection (without age limitations) that helps us better understand the impact of HIV/AIDS on older people is urgently needed. HIV/AIDS information, education and prevention activities as well as treatment services should apply to all ages.

Numerous studies have found that most adult children with AIDS return home to die. Wives, mothers, aunts, sisters, sisters-in-law and grandmothers take on the bulk of the care. Then, in many cases, these women take on the care of the orphaned children. Governments, nongovernmental organizations and private industry need to address the financial, social and training needs of older people who care for family members and neighbours who are infected and raise child survivors, some of whom themselves are also infected (WHO, 2002). 
which could have been prevented in the first place (e.g., lung cancer, diabetes and peripheral vascular disease) or a degenerative illness (e.g., dementia). The likelihood of experiencing serious cognitive and physical disabilities dramatically increases in very old age. Significantly, adults over the age of 80 are the fastest growing age group worldwide.

But disabilities associated with ageing and the onset of chronic disease can be prevented or delayed. For example, as mentioned on page 18 , there has been a significant decline over the last 20 years in age-specific disability rates in the U.S.A (see Figure 11), England, Sweden and other developed countries.

Figure 10 shows the actual decline in disabilities among older Americans between 1982 and 1999 compared to the projected numbers if rates of disability had remained stable over that time period.
Some of this decline is likely due to increased education levels, improved standards of living and better health in the early years. The adoption of positive lifestyle behaviours is also a factor. As already mentioned, choosing not to smoke and making modest increases in physical activity levels can significantly reduce one's risk for heart disease and other illnesses. Supportive changes in the community are also important, both in terms of preventing disabilities and reducing the restrictions that people with disabilities often face. In addition, impressive progress in the management of chronic conditions has been observed, including new techniques for early diagnosis and treatment, as well as long-term management of chronic diseases, such as hypertension and arthritis. Recent studies have also emphasized that the increasing use of aids - from simple personal aids, such as canes, walkers, handrails, to technologies aimed at the population as a whole, such as telephones - may reduce

\section{Figure 11. Numbers of chronically disabled Americans aged 65 and over (in millions), \\ 1982 to 1999 , actual and projected numbers}

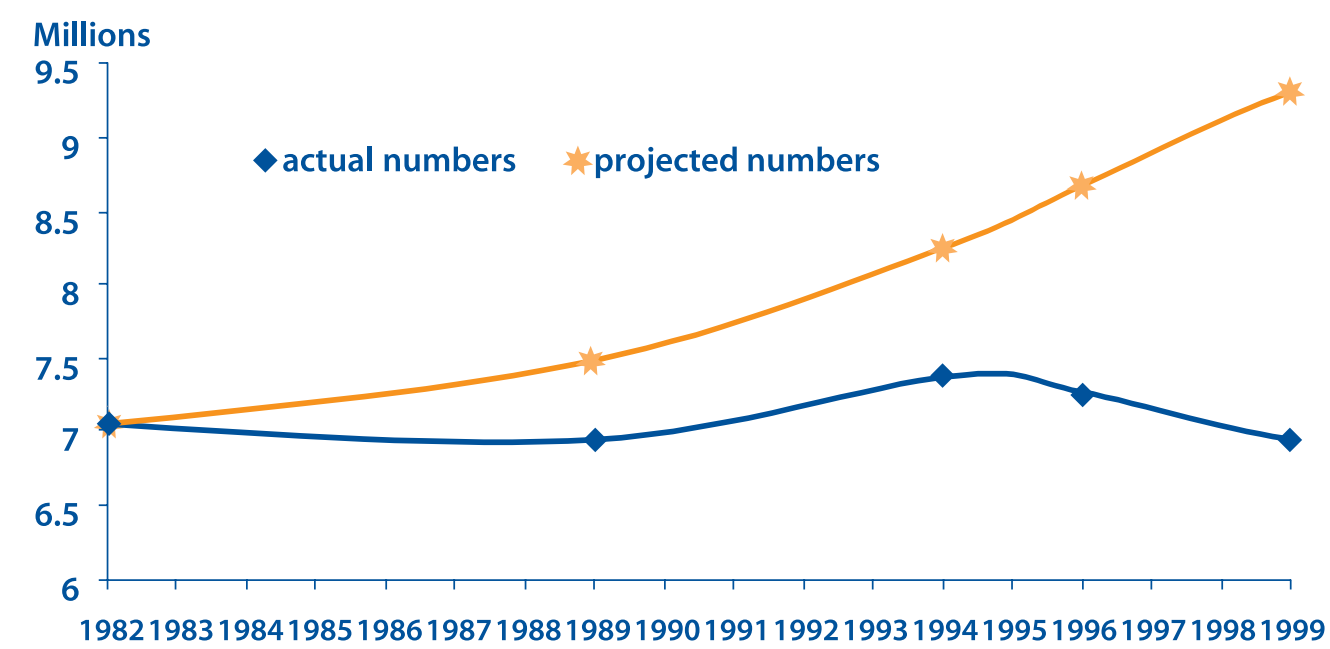

Total number of older people in the USA

1982:26.9; 1994:33.1; 1999:35.3 (millions)

Source: Manton and Gu, 2001 
dependence among disabled people. In the USA the use of such aids by dependent older people increased from 76 percent in 1984 to over 90 percent in 1999 (Cutler, 2001).

\section{Vision and Hearing}

Other common age-related disabilities include vision and hearing losses. Worldwide, there are currently 180 million people with visual disability, up to 45 million of whom are blind. Most of these are older people, as visual impairment and blindness increase sharply with age. Overall, approximately four percent of persons aged 60 years and above are thought to be blind, and 60 percent of them live in Sub-Saharan Africa, China and India. The major age-related causes of blindness and visual disability include cataracts (nearly 50 percent of all blindness), glaucoma, macular degeneration and diabetic retinopathy (WHO, 1997).

There is an urgent need for policies and programmes designed to prevent visual impairment and to increase appropriate eye care services, particularly in developing countries. In all countries, corrective lenses and cataract surgery should be accessible and affordable for older people who need them.

Hearing impairment leads to one of the most widespread disabilities, particularly in older people. It is estimated that worldwide over 50 percent of people aged 65 years and over have some degree of hearing loss (WHO, 2002a). Hearing loss can cause difficulties with communication. This, in turn can lead to frustration, low self-esteem, withdrawal and social isolation (Pal, 1974, Wilson, 1999).
Policies and programmes need to be in place to reduce and eventually eliminate avoidable hearing impairment and to help people with hearing loss obtain hearing aids. Hearing loss may be prevented by avoiding exposure to excessive noise and the use of potentially damaging drugs and by early treatment of diseases leading to hearing loss, such as middle ear infections, diabetes and possibly hypertension. Hearing loss can sometimes be treated, especially if the cause is in the ear canal or middle ear. Most often, however, the disability is reduced by amplification of sounds, usually by using a hearing aid.

\section{An Enabling Environment}

As populations around the world live longer, policies and programmes that help prevent and reduce the burden of disability in old age are urgently needed in both developing and developed countries. One useful way to look at decision-making in this area is to think about enablement instead of disablement. Disabling processes increase the needs of older people and lead to isolation and dependence. Enabling processes restore function and expand the participation of older people in all aspects of society.

A variety of sectors can enact "age-friendly" policies that prevent disability and enable those who have disabilities to fully participate in community life. Here are some examples of enabling programmes, environments and policies in a variety of sectors:

- barrier-free workplaces, flexible work hours, modified work environments and part-time work for people who experience disabilities as they age or are required to care for others with disabilities (private industry and employers) 
- well-lit streets for safe walking, accessible public toilets and traffic lights that give people more time to cross the street (local governments)

- exercise programmes that help older people maintain their mobility or recover the leg strength they need to be mobile (recreation services and nongovernmental agencies)

- life-long learning and literacy programmes (education sector and nongovernmental organizations)

- hearing aids or instruction in sign language that enables older people who are hard of hearing to continue to communicate with others (social services and nongovernmental organizations)

- barrier-free access to health centres, rehabilitation programmes and cost-effective procedures such as cataract surgery and hip replacements (health sector)

- credit schemes and access to small business and development opportunities so that older people can continue to earn a living (governments and international agencies).

Changing the attitudes of health and social service providers is paramount to ensuring that their practices enable and empower individuals to remain as autonomous and independent as possible for as long as possible. Professional caregivers need to respect older people's dignity at all times and to be careful to avoid premature interventions that may unintentionally induce the loss of independence.
Researchers need to better define and standardize the tools used to assess ability and disability and to provide policy makers with additional evidence on key enabling processes in the broader environment, as well as in medicine and health. Careful attention needs to be paid to gender differences in these analyses.

\section{Challenge 3: Providing Care for Ageing Populations}

As populations age, one of the greatest challenges in health policy is to strike a balance among support for self-care (people looking after themselves), informal support (care from family members and friends) and formal care (health and social services). Formal care includes both primary health care (delivered mostly at the community level) and institutional care (either in hospitals or nursing homes). While it is clear that most of the care individuals need is provided by themselves or their informal caregivers, most countries allot their financial resources inversely, i.e., the greatest share of expenditure is on institutional care.

All over the world, family members, friends and neighbours (most of whom are women) provide the bulk of support and care to older adults that need assistance. Some policy makers fear that providing more formal care services will lessen the involvement of families. Studies show that this is not the case. When appropriate formal services are provided, informal care remains the key partner (WHO, 2000c). Of concern though are recent demographic trends in a large number of countries indicating the increase in the proportion of childless women, changes in divorce and marriage patterns and the overall much smaller number of children of future cohorts of older people, all contributing to a shrinking pool of family support (Wolf, 2001). 
Formal care through health and social service systems needs to be equally accessible to all. In many countries older people who are poor and who live in rural areas have limited or no access to needed health care. A decline in public support for primary health care services in many areas has put increased financial and intergenerational strain on older people and their families.

Most older persons in need of care prefer to be cared for in their own homes. But caregivers (who are often older people) must be supported if they are to continue to provide care without becoming ill themselves. Above all, they need to be well informed about the condition they are faced with and how it is likely to progress, and about how to obtain the support services that are available. Visiting nurses, home care, peer support programmes, rehabilitation services, the provision of assistive devices (ranging from basic devices such as a hearing aid to more sophisticated ones, such as an electronic alarm system), respite care and adult day care are all important services that enable informal caregivers to continue to provide care to individuals who require help, whatever their age. Other forms of support include training, income security (e.g., social security coverage and pensions), help with housing adjustments that enable families to look after people who are disabled and disbursements to help cover caring costs.

As the proportion of older people increases in all countries, living at home into very old age with help from family members will become increasingly common. Home care and community services to assist informal caregivers need to be available to all, not just to those who know about them or can afford to pay for them.

\section{Figure 12. Sex ratios by world regions, age 60 years and over, 2002 and 2020}

\section{Number of men per 1,000 women}

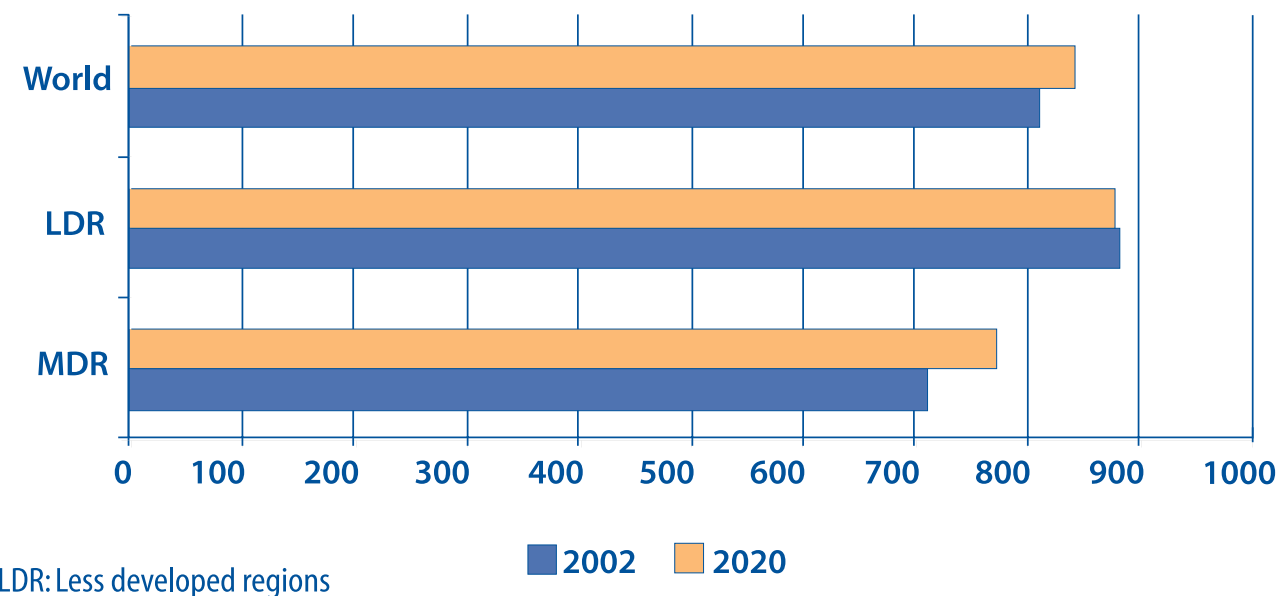

MDR:More developed regions

Medium variant fertility

Source:UN, 2001

Sex ratios for populations age 60 and over reflect the larger proportion of women than men in all regions of the world, particularly in the more developed regions. 
Professional caregivers also need training and practice in enabling models of care that recognize older people's strengths and empower them to maintain even small measures of independence when they are ill or frail. Paternalistic or disrespectful attitudes by professionals can have a devastating effect on the self-esteem and independence of older people who require services.

Information and education about active ageing needs to be incorporated into curricula and training programmes for all health, social service and recreation workers as well as city planners and architects. Basic principles and approaches in old-age care should be mandatory in the training of all medical and nursing students as well as other health professions.

\section{Challenge 4: The Feminization of Ageing}

Women live longer than men almost everywhere. This is reflected in the higher ratio of women versus men in older age groups. For example, in 2002, there were 678 men for every 1,000 women aged 60 plus in Europe. In less developed regions, there were 879 men per 1,000 women (See Figure 12). Women make up approximately two-thirds of the population over age 75 in countries such as Brazil and South Africa. While women have the advantage in length of life, they are more likely than men to experience domestic violence and discrimination in access to education, income, food, meaningful work, health care, inheritances, social security measures and political power. These cumulative disadvantages mean that women are more likely than

\section{Figure 13. Population pyramid for Japan in 2002 and 2025}

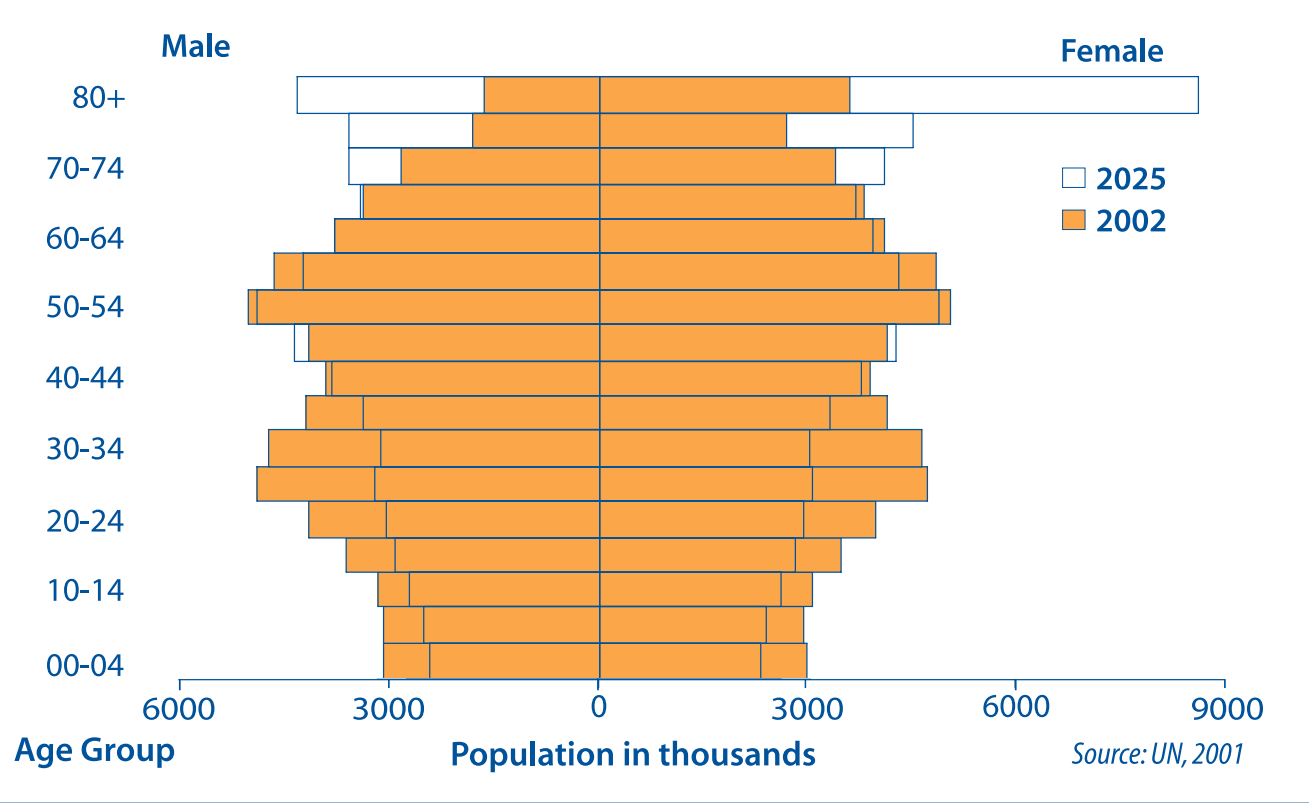

In contrast to the pyramid form, the apanese population structure has changed due to population ageing towards a cone shape. By 2025, the shape will be similar to an up-side-down pyramid, with persons age 80 and over accounting for the largest population group. The feminization of old age is highly visible. 
men to be poor and to suffer disabilities in older age. Because of their second-class status, the health of older women is often neglected or ignored. In addition, many women have low or no incomes because of years spent in unpaid caregiving roles. The provision of family care is often achieved at the detriment of female caregivers' economic security and good health in later life.

Women are also more likely than men to live to very old age when disabilities and multiple health problems are more common. At age 80 and over, the world average is below 600 men for every 1,000 women. In the more developed regions women age 80 and over outnumber men by more than two to one (see the example of Japan in Figure 13).

Because of women's longer life expectancy and the tendency of men to marry younger women and to remarry if their spouses die, female widows dramatically outnumber male widowers in all countries. For example, in the Eastern European countries in economic transition over 70 percent of women age 70 and over are widows (Botev, 1999).

Older women who are alone are highly vulnerable to poverty and social isolation. In some cultures, degrading and destructive attitudes and practices around burial rights and inheritance may rob widows of their property and possessions, their health and independence and, in some cases, their very lives.

\section{Challenge 5: Ethics and Inequities}

As populations age, a range of ethical considerations comes to the fore. They are often linked to age discrimination in resource allocation, issues related to the end of life and a host of dilemmas linked to long-term care and the human rights of poor and disabled older citizens. Scientific advancements and modern medicine have led to many ethical questions related to genetic research and manipulation, biotechnology, stem cell research and the use of technology to sustain life while compromising quality of life. In all cultures, consumers need to be fully informed about false claims of "anti-ageing" products and programmes that are ineffective or harmful. They need protection from fraudulent marketing and financing schemes, especially as they grow older.

Societies that value social justice must strive to ensure that all policies and practices uphold and guarantee the rights of all people, regardless of age. Advocacy and ethical decision-making must be central strategies in all programmes, practices, policies and research on ageing.

Older age often exacerbates other pre-existing inequalities based on race, ethnicity or gender. While women are universally disadvantaged in terms of poverty, men have shorter life expectancies in most countries. The exclusion and impoverishment of older women and men is often a product of structural inequities in both developing and developed countries. Inequalities experienced in earlier life in access to education, employment and health care, as well as those based on gender and race have a critical bearing on status and well being in old age. For older people who are poor, the consequences of these earlier experiences 
are worsened through further exclusion from health services, credit schemes, income-generating activities and decision-making. Inequities in care occur when small and comparatively well off portions of the ageing population, particularly those in developing countries, consume a disproportionately high amount of public resources for their care.

In many cases, the means for older people to achieve dignity and independence, receive care and participate in civic affairs are very limited. These conditions are often worse for older people living in rural areas, in countries in transition and in situations of conflict or humanitarian disasters.

In all regions of the world, relative wealth and poverty, gender, ownership of assets, access to work and control of resources are key factors in socioeconomic status. Recent World Bank data reveal that in many developing countries well over half of the population lives on less than two purchasing power parity (PPP) dollars per day (see Table 4).

It is well known that socioeconomic status and health are intimately related. With each step up the socioeconomic ladder, people live longer, healthier lives (Wilkinson, 1996). In recent years, the gap between rich and poor and subsequent inequalities in health status has been increasing in countries in all parts of the world (Lynch et al, 2000). Failure to address this problem will have serious consequences for the global economy and social order, as well as for individual societies and people of all ages.

Table 4. Percentage of the population below international poverty lines in countries with a population approaching or above 100 million in the year 2000

\begin{tabular}{|l|r|r|r|}
\hline Countries & $\begin{array}{r}\text { Population } \\
\text { (millions)\# }\end{array}$ & $\begin{array}{r}\text { Percentage with } \\
\text { <1dollar/day* }\end{array}$ & $\begin{array}{r}\text { Percentage with } \\
<2 \text { dollar/day* }\end{array}$ \\
\hline China & 1.275 & 18.5 & 53.7 \\
\hline India & 1.008 & 44.2 & 86.2 \\
\hline Indonesia & 212 & 7.7 & 55.3 \\
\hline Brazil & 170 & 9.0 & 25.4 \\
\hline Russian Federation & 145 & 7.1 & 25.1 \\
\hline Pakistan & 141 & 31.0 & 84.7 \\
\hline Bangladesh & 137 & 29.1 & 77.8 \\
\hline Nigeria & 113 & 70.2 & 90.8 \\
\hline Mexico & 98 & 12.2 & 34.8 \\
\hline
\end{tabular}

*adjusted for purchasing power

Source:World Bank,2001, \#Source:UN, 2001 


\section{Challenge 6: The Economics of an Ageing Population}

Perhaps more than anything else, policy makers fear that rapid population ageing will lead to an unmanageable explosion in health care and social security costs. While there is no doubt that ageing populations will increase demands in these areas, there is also evidence that innovation, cooperation from all sectors, planning ahead and making evidence-based, culturally-appropriate policy choices will enable countries to successfully manage the economics of an ageing population.

Research in countries with aged populations has shown that ageing per se is not likely to lead to "health care costs that are spiraling out of control", for two reasons.

First, according to OECD data, the major causes of escalating health care costs are related to circumstances that are unrelated to the demographic ageing of a given population. Inefficiencies in care delivery, building too many hospitals, payment systems that encourage long hospital stays, excessive numbers of medical interventions and the inappropriate use of high cost technologies are the key factors in escalations in health care costs. For example, in the United States and other OECD countries, new technologies were sometimes rapidly introduced and used where alternative and less expensive procedures already existed, and for which the marginal effectiveness was relatively low (Jacobzone and Oxley, 2002). There appears to be considerable scope for policy makers to address these issues and improve the effectiveness of health care.
Second, the costs of long-term care can be managed if policies and programmes address prevention and the role of informal care. Policies and health promotion programmes that prevent chronic diseases and lessen the degree of disability among older citizens enable them to live independently longer. Another major factor is the capacity and willingness of families to provide care and support for older family members. This will depend to a large extent on the rates of female participation in the labour force and on workplace and public policies that recognize and support the caregiving role.

In many countries, the bulk of spending is on curative medicine. Care for chronic conditions leads to an improved quality of life; however, it is always preferable if those conditions could be prevented or delayed until very late in life. Decision makers need to evaluate whether such outcomes can be achieved through policies that address the broad determinants of active ageing, such as interventions to prevent injuries, improve diets and physical activity, increase literacy or increase employment.

Ultimately, the level of funding allocated to the health system is a social and political choice with no universally applicable answer. However, the WHO suggests that it is better to make pre-payments on health care as much as possible, whether in the form of insurance, taxes or social security. The principle of "fair financing" ensures equity of access regardless of age, sex or ethnicity and that the financial burden is shared in a fair way (WHO, 2000a). 
A second major concern to policy-makers is the demand that an ageing population may put on social security systems. Alarmists point to the growing proportion of the "dependent" population that has retired from the formal labour force. The idea that everyone over age 60 is dependent is, however, a false assumption. Many people continue to work in the formal labour market in later life or would choose to do so if the opportunity existed. Many others continue to contribute to the economy through informal work and voluntary activities, as well as intergenerational exchanges of cash and family support. For example, older people who look after grandchildren allow younger adults to participate in the labour market.

An ageing population provides other advantages to the overall economy. Nations with declining working-age populations will be able to draw on older experienced workers and industries will be able to grow as they serve the needs of older consumers.

Global ageing does require governments and the private sector to address the challenges to social security and pension systems. A balanced approach to the provision of social protection and economic goals suggests that societies who are willing to plan can afford to grow old. Labour market policies (for example, incentives for early retirement and mandatory retirement practices) have a more dramatic impact on a nation's ability to provide social protection in old age than demographic ageing per se. The goal must be to ensure adequate living standards for people as they grow older, while recognizing and harnessing their skills and experience and encouraging harmonious intergenerational transfers.

\section{Challenge 7: Forging a New Paradigm}

Traditionally, old age has been associated with retirement, illness and dependency. Policies and programmes that are stuck in this outdated paradigm do not reflect reality. Indeed, most people remain independent into very old age. Especially in developing countries, many people over age 60 continue to participate in the labour force. Older people are active in the informal work sector (e.g., domestic work and small scale, self-employed activities) although this is often not recognized in labour market statistics. Older people's unpaid contributions in the home (such as looking after children and people who are ill) allow younger family members to engage in paid labour. In all countries, the voluntary activities of older people provide an important economic and social contribution to society.

\section{It is time for a new paradigm, one that views older people as active participants in an age-integrated society and as active contributors as well as beneficiaries of development.}

This includes recognition of the contributions of older people who are ill, frail and vulnerable and championing their rights to care and security.

This paradigm takes an intergenerational approach that recognizes the importance of relationships and support among and between family members and generations. It reinforces "a society for all ages" - the central focus of the 1999 United Nations International Year of Older Persons. 
The new paradigm also challenges the traditional view that learning is the business of children and youth, work is the business of midlife and retirement is the business of old age. The new paradigm calls for programmes that support learning at all ages and allow people to enter or leave the labour market in order to assume caregiving roles at different times over the life course. This approach supports intergenerational solidarity and provides increased security for children, parents and people in their old age.
Older people themselves and the media must take the lead in forging a new, more positive image of ageing. Political and social recognition of the contributions that older people make and the inclusion of older men and women in leadership roles will support this new image and help de-bunk negative stereotypes. Educating young people about ageing and paying careful attention to upholding the rights of older people will help to reduce and eliminate discrimination and abuse. 


\section{The Policy Response}

The ageing of the population is a global phenomenon that demands international, national, regional and local action. In an increasingly inter-connected world, failure to deal with the demographic imperative and rapid changes in disease patterns in a rational way in any part of the world will have socioeconomic and political consequences everywhere.

Ultimately, a collective approach to ageing and older people will determine how we, our children and our grandchildren will ex perience life in later years.
The policy framework for active ageing shown below is guided by the United Nations Principles for Older People (the outer circle). These are independence, participation, care, self-fulfillment and dignity. Decisions are based on an understanding of how the determinants of active ageing influence the way that individuals and populations age.

The policy framework requires action on three basic pillars:

Health. When the risk factors (both environmental and behavioural) for chronic diseases and functional decline are kept low while the protective factors are kept high, people will enjoy both a longer quantity and quality of

Figure 14. The three pillars of a policy framework for Active Ageing

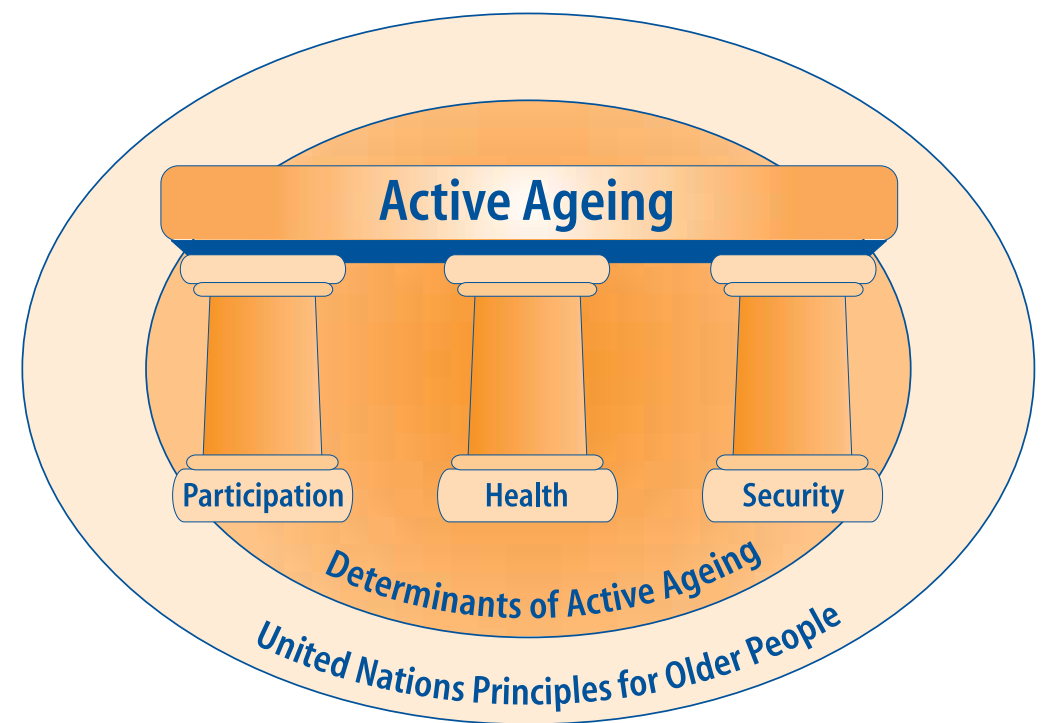


life; they will remain healthy and able to manage their own lives as they grow older; fewer older adults will need costly medical treatment and care services.

For those who do need care, they should have access to the entire range of health and social services that address the needs and rights of women and men as they age.

Participation. When labour market, employment, education, health and social policies and programmes support their full participation in socioeconomic, cultural and spiritual activities, according to their basic human rights, capacities, needs and preferences, people will continue to make a productive contribution to society in both paid and unpaid activities as they age.

Security. When policies and programmes address the social, financial and physical security needs and rights of people as they age, older people are ensured of protection, dignity and care in the event that they are no longer able to support and protect themselves. Families and communities are supported in efforts to care for their older members.

\section{Intersectoral Action}

Attaining the goal of active ageing will require action in a variety of sectors in addition to health and social services, including education, employment and labour, finance, social security, housing, transportation, justice and rural and urban development. While it is clear that the health sector does not have direct responsibility for policies in all of these other sectors, they belong in the broadest sense within the scope of public health because they support the goals of improved health through intersectoral action. This kind of an approach stresses the importance of the numerous different public health partners and reinforces the role of the health sector as a catalyst for action (Yach, 1996).

Furthermore, all policies need to support intergenerational solidarity and include specific targets to reduce inequities between women and men and among different subgroups within the older population. Particular attention needs to be paid to older people who are poor and marginalized, and who live in rural areas.

An active ageing approach seeks to eliminate age discrimination and recognize the diversity of older populations. Older people and their caregivers need to be actively involved in the planning, implementation and evaluation of policies, programmes and knowledge development activities related to active ageing.

\section{Key Policy Proposals}

The following policy proposals are designed to address the three pillars of active ageing: health, participation and security. Some are broad and encompass all age groups while others are targeted specifically to those approaching old age and/or older people themselves. 


\section{Health}

\subsection{Prevent and reduce the burden of ex cess disabilities, chronic disea se and prema ture morta lity.}

- Goals and targets. Set gender-specific, measurable targets for improvements in health status among older people and in the reduction of chronic diseases, disabilities and premature mortality as people age.

\section{- Economic influences on health. Enact} policies and programmes that address the economic factors that contribute to the onset of disease and disabilities in later life (i.e., poverty, income inequities and social exclusion, low literacy levels, lack of education). Give priority to improving the health status of poor and marginalized population groups.

\section{- Prevention and effective treatments.} Make screening services that are proven to be effective, available and affordable to women and men as they age. Make effective, cost-efficient treatments that reduce disabilities (such as cataract removal and hip replacements) more accessible to older people with low incomes.

- Age-friendly, safe environments. Create age-friendly health care centres and standards that help prevent the onset or worsening of disabilities. Prevent injuries by protecting older pedestrians in traffic, making walking safe, implementing fall prevention programmes, eliminating hazards in the home and providing safety advice. Stringently enforce occupational safety standards that protect older workers from injury. Modify formal and informal work environments so that people can continue to work productively and safely as they age.

- Hearing and vision. Reduce avoidable hearing impairment through appropriate prevention measures and support access to hearing aids for older people who have hearing loss. Aim to reduce and eliminate avoidable blindness by 2020 (WHO, 1997). Provide appropriate eye care services for people with age-related visual disabilities. Reduce inequities in access to corrective glasses for ageing women and men.

- Barrier-free living. Develop barrier-free housing options for ageing people with disabilities. Work to make public buildings and transportation accessible for all people with disabilities. Provide accessible toilets in public places and workplaces.

- Quality of life. Enact policies and programmes that improve the quality of life of people with disabilities and chronic illnesses. Support their continuing independence and interdependence by assisting with changes in the environment, providing rehabilitation services and community support for families, and increasing affordable access to effective assistive devices (e.g., corrective eyeglasses, walkers). 
- Social support. Reduce risks for loneliness and social isolation by supporting community groups run by older people, traditional societies, self-help and mutual aid groups, peer and professional outreach programmes, neighbourhood visiting, telephone support programmes, and family caregivers. Support intergenerational contact and provide housing in communities that encourage daily social interaction and interdependence among young and old.

- HIV and AIDS. Remove the age limitation on data collection related to HIV/AIDS. Assess and address the impact of HIV/AIDS on older people, including those who are infected and those who are caring for others who are infected and/or for AIDS orphans.

- Mental health. Promote positive mental health throughout the life course by providing information and challenging stereotypical beliefs about mental health problems and mental illness.

- Clean environments. Put policies and programmes in place that ensure equal access for all to clean water, safe food and clean air. Minimize exposure to pollution throughout the life course, particularly in childhood and old age.

\subsection{Reduce risk factors a ssociated with major diseases and increase factors that protect health throughout the life course.}

- Tobacco. Take comprehensive action at local, national and international levels to control the marketing and use of tobacco products. Provide older people with help to quit smoking.
- Physical activity. Develop culturally appropriate, population-based information and guidelines on physical activity for older men and women. Provide accessible, pleasant and affordable opportunities to be active (e.g., safe walking areas and parks). Support peer leaders and groups that promote regular, moderate physical activity for people as they age. Inform and educate people and professionals about the importance of staying active as one grows older.

- Nutrition. Ensure adequate nutrition throughout the life course, particularly in childhood and among women in the reproductive years. Ensure that national nutrition policies and action plans recognize older persons as a potentially vulnerable group. Include special measures to prevent malnutrition and ensure food security and safety as people age.

- Healthy eating. Develop culturally appropriate, population-based guidelines for healthy eating for men and women as they age. Support improved diets and healthy weights in older age through the provision of information (including information specific to the nutrition needs of older people), education about nutrition at all ages, and food policies that enable women, men and families to make healthy food choices.

- Oral health. Promote oral health among older people and encourage women and men to retain their natural teeth for as long as possible. Set culturally appropriate policy goals for oral health and provide appropriate oral health promotion programmes and treatment services during the life course. 
- Psychological factors. Encourage and enable people to build self-efficacy, cognitive skills such as problem-solving, prosocial behaviour and effective coping skills throughout the life course. Recognize and capitalize on the experience and strengths of older people while helping them improve their psychological well being.

- Alcohol and drugs. Determine the extent of the use of alcohol and drugs by people as they age and put practices and policies in place to reduce misuse and abuse.

- Medications. Increase affordable access to essential safe medications among older people who need them but cannot afford them. Put practices and policies in place to reduce inappropriate prescribing by health professionals and other health advisors. Inform and educate people about the wise use of medications.

- Adherence. Undertake comprehensive measures to better understand and correct poor adherence to therapies, which severely compromise treatment effectiveness, particularly in relation to long-term therapies.

\subsection{Develop a continuum of affordable,} accessible, high quality and agefriendly health and social services that address the needs and rights of women and men as they age.

\section{- A continuum of care throughout the}

life course. Taking into consideration their opinions and preferences, provide a continuum of care for women and men as they grow older. Re-orient current systems that are organized around acute care to provide a seamless continuum of care that includes health promotion, disease prevention, the appropriate treatment of chronic diseases, the equitable provision of community support and dignified long-term and palliative care through all the stages of life.

- Affordable, equitable access. Ensure affordable equitable access to quality primary health care (both acute and chronic), as well as long-term care services for all.

- Informal ca regivers. Recognize and address gender differences in the burden of caregiving and make a special effort to support caregivers, most of whom are older women who care for partners, children, grandchildren and others who are sick or disabled. Support informal caregivers through initiatives such as respite care, pension credits, financial subsidies, training and home care nursing services. Recognize that older caregivers may become socially isolated, financially disadvantaged and sick themselves, and attend to their needs.

- Formal caregivers. Provide paid caregivers with adequate working conditions and remuneration, with special attention to those who are unskilled and have low social and professional status (most of whom are women). 
- Mental health services. Provide comprehensive mental health services for men and women as they age, ranging from mental health promotion to treatment services for mental illness, rehabilitation and re-integration into the community as required. Pay special attention to increased depression and suicidal tendencies due to loss and social isolation. Provide quality care for older people with dementia and other neurological and cognitive problems in their homes and in residential facilities when appropriate. Pay special attention to ageing people with long-term intellectual disabilities.

\section{- Coordinated ethical systems of care.}

Eliminate age discrimination in health and social service systems. Improve the coordination of health and social services and integrate these systems when feasible. Set and maintain appropriate standards of care for ageing persons through regulatory mechanisms, guidelines, education, consultation and collaboration.

- Ia trogenesis. Prevent iatrogenesis (disease and disability that is induced by the process of diagnosis or treatment). Establish adequate systems for preventing adverse drug reactions with a special focus on old age. Raise awareness of the relative risks and benefits of modern therapies among health professionals and the public at large.

\section{- Ageing at home and in the community.} Provide policies, programmes and services that enable people to remain in their homes as they grow older, with or without other family members according to their circumstances and preferences. Support families that include older people who need care in their households. Provide help with meals and home maintenance, and at-home nursing support when it is required.

- Partnerships and quality care. Provide a comprehensive approach to long-term care (by informal and formal caregivers) that stimulates collaboration between the public and private sectors and involves all levels of government, civil society and the not-for-profit sector. Ensure high quality standards and stimulating environments in residential care facilities for men and women who require this care, as they grow older.

\subsection{Provide training and education to caregivers.}

- Informal caregivers. Provide family members, peer counsellors and other informal caregivers with information and training on how to care for people as they grow older. Support older healers who are knowledgeable about traditional and complementary medicines while also assessing their training needs.

- Formal caregivers. Educate health and social service workers in enabling models of primary health care and long-term care that recognize the strengths and contributions of older people. Incorporate modules on active ageing in medical and health curricula at all levels. Provide specialist education in gerontology and geriatrics for medical, health and social service professionals.

Inform all health and social service professionals about the process of ageing and 
ways to optimize active ageing among individuals, communities and population groups. Provide incentives and training for health and social service professionals to support self-care and counsel healthy lifestyle practices among men and women as they age. Increase the awareness and sensitivity of all health professionals and community workers of the importance of social networks for well being in old age. Train health promotion workers to identify older people who are at risk for loneliness and social isolation.

\section{Participation}

\subsection{Provide education and learning op- portunities throughout the life course.}

\section{- Basic education and health literacy.}

Make basic education available to all across the life course. Aim to achieve literacy for all. Promote health literacy by providing health education throughout the life course. Teach people how to care for themselves and each other as they get older. Educate and empower older people on how to effectively select and use health and community services.

- Lifelong learning. Enable the full participation of older people by providing policies and programmes in education and training that support lifelong learning for women and men as they age. Provide older people with opportunities to develop new skills, particularly in areas such as information technologies and new agricultural techniques.
2.2 Recognize and enable the active participation of people in economic development activities, formal and informal work and voluntary activities as they age, according to their individual needs, preferences and capacities.

- Poverty reduction and income generation. Include older people in the planning, implementation and evaluation of social development initiatives and efforts to reduce poverty. Ensure that older people have the same access to development grants, income-generation projects and credit as younger people do.

- Formal work. Enact labour market and employment policies and programmes that enable the participation of people in meaningful work as they grow older, according to their individual needs, preferences and capacities (e.g., the elimination of age discrimination in the hiring and retention of older workers). Support pension reforms that encourage productivity, a diverse system of pension schemes and more flexible retirement options (e.g., gradual or partial retirement).

- Informal work. Enact policies and programmes that recognize and support the contribution that older women and men make in unpaid work in the informal sector and in caregiving in the home.

- Voluntary activities. Recognize the value of volunteering and expand opportunities to participate in meaningful volunteer activities as people age, especially those who want to volunteer but cannot because of health, income, or transportation restrictions. 


\subsection{Encourage people to participate fully} in fa mily community life, as they grow older.

- Transportation. Provide accessible, affordable public transportation services in rural and urban areas so that older people (especially those with compromised mobility) can participate fully in family and community life.

- Leadership. Involve older people in political processes that affect their rights. Include older women and men in the planning, implementation and evaluation of locally based health and social service and recreation programmes. Include older people in prevention and education efforts to reduce the spread of HIV/AIDS. Involve older people in efforts to develop research agendas on active ageing, both as advisors and as investigators.

- A society for all ages. Provide greater flexibility in periods devoted to education, work and caregiving responsibilities throughout the life course. Develop a range of housing options for older people that eliminate barriers to independence and interdependence with family members, and encourage full participation in community and family life. Provide intergenerational activities in schools and communities. Encourage older people to become role models for active ageing and to mentor young people. Recognize and support the important role and responsibilities of grandparents. Foster collaboration among nongovernmental organizations that work with children, youth and older people.
- A positive image of ageing. Work with groups representing older people and the media to provide realistic and positive images of active ageing, as well as educational information on active ageing. Confront negative stereotypes and ageism.

\section{- Reduce inequities in participation} by women. Recognize and support the important contribution that older women make to families and communities through caregiving and participation in the informal economy. Enable the full participation of women in political life and decision-making positions as they age. Provide education and lifelong learning opportunities to women as they age, in the same way that they are provided to men.

\section{- Support organizations representing} older people. Provide in-kind and financial support and training for members of these organizations so that they can advocate, promote and enhance the health, security and full participation of older women and men in all aspects of community life.

\section{Security}

\subsection{Ensure the protection, sa fety and dig-} nity of older people by addressing the social, financial and physical security rights and needs of people as they age.

- Social security. Support the provision of a social safety net for older people who are poor and alone, as well as social security initiatives that provide a steady and adequate stream of income during old age. Encourage young adults to prepare for old age in their health, social and financial practices. 
- HIV/AIDS. Support the social, economic and psychological well being of older people who care for people with HIV/AIDS and take on surrogate parenting roles for orphans of AIDS. Provide in-kind support, affordable health care and loans to older people to help them meet the needs of children and grandchildren affected by HIV/AIDS.

- Consumer protection. Protect consumers from unsafe medications and treatments, and unscrupulous marketing practices, particularly in older age.

- Social justice. Ensure that decisions being made concerning care in older age are based on the rights of older people and guided by the UN Principles for Older Persons. Uphold older persons' rights to maintain independence and autonomy for the longest period of time possible.

- Shelter. Explicitly recognize older people's right to and need for secure, appropriate shelter, especially in times of conflict and crisis. Provide housing assistance for older people and their families when required (paying special attention to the circumstances of those who live alone) through rent subsidies, cooperative housing initiatives, support for housing renovations, etc.

- Crises. Uphold the rights of older people during conflict. Specifically recognize and act on the need to protect older people in emergency situations (e.g., by providing transportation to relief centres to those who cannot walk there). Recognize the contribu- tion that older people can make to recovery efforts in the aftermath of an emergency and include them in recovery initiatives.

- Elder abuse. Recognize elder abuse (physical, sexual, psychological, financial and neglect) and encourage the prosecution of offenders. Train law enforcement officers, health and social service providers, spiritual leaders, advocacy organizations and groups of older people to recognize and deal with elder abuse. Increase awareness of the injustice of elder abuse through public information and awareness campaigns. Involve the media and young people, as well as older people in these efforts.

\subsection{Reduce inequities in the security rights and needs of older women.}

- Enact legislation and enforce laws that protect widows from the theft of property and possessions and from harmful practices such as health-threatening burial rituals and charges of witchcraft.

- Enact legislation and enforce laws that protect women from domestic and other forms of violence as they age.

- Provide social security (income support) for older women who have no pensions or meager retirement incomes because they have worked all or most of their lives in the home or informal sector. 


\section{WHO and Ageing}

In 1995 when WHO renamed its "Health of the Elderly Programme"to "Ageing and Health", it signaled an important change in orientation. Rather than compartmentalizing older people, the new name embraced a life course perspective: we are all ageing and the best way to ensure good health for future cohorts of older people is by preventing diseases and promoting health throughout the life course. Conversely, the health of those now in older age can only be fully understood if the life events they have gone through are taken into consideration.

The aim of the Ageing and Health Programme has been to develop policies that ensure "the attainment of the best possible quality of life for as long as possible, for the largest possible number of people."For this to be achieved,WHO is required to advance the knowledge base of gerontology and geriatric medicine through research and training efforts. Emphasis is needed on fostering interdisciplinary and intersectoral initiatives, particularly those directed at developing countries faced with unprecedented rapid rates of population ageing within a context of prevailing poverty and unsolved infrastructure problems. In addition the Programme highlighted the importance of:

- adopting community-based approaches by emphasizing the community as a key setting for interventions

- respecting cultural contexts and influences

- recognizing the importance of gender differences

- strengthening intergenerational links

- respecting and understanding ethical issues related to health and well being in old age.

The International Year of Older Persons (1999) was a landmark in the evolution of the WHO's work on ageing and health. That year, the World Health Day theme was "active ageing makes the difference" and the "Global Movement for Active Ageing" was launched by the WHO Director-General, Dr Gro Harlem Brundtland. At this occasion, Dr Brundtland stated: Maintaining health and quality of life across the lifespan will do much towards building fulfilled lives, a harmonious intergenerational community and a dynamic economy.WHO is committed to promoting ActiveAgeing as an indispensable component of all development programmes.

In 2000 , the name of the WHO programme was changed again to "Ageing and Life Course"to reflect the importance of the life-course perspective. The multi-focus of the previous programme and the emphasis on developing activities with multiple partners from all sectors and several disciplines have been maintained. A further refinement of the ' active ageing' concept has been added and translated into all the programme activities, including research and training, information dissemination, advocacy and policy development.

In addition to the Ageing and Life Course Programme at WHO Headquarters, each of the six WHO Regional Offices have their own Adviser on Ageing in order to address specific issues from a regional perspective. 


\section{International Collaboration}

With the launch of the International Plan of Action on Ageing, the 2002 World Assembly on Ageing marks a turning point in addressing the challenges and celebrating the triumphs of an ageing world. As we embark on the implementation phase, cross-national, regional and global sharing of research and policy options will be critical. Increasingly, member states, nongovernmental organizations, academic institutions and the private sector will be called upon to develop age-sensitive solutions to the challenges of an ageing world. They will need to take into consideration the consequences of the epidemiological transition, rapid changes in the health sector, globalization, urbanization, changing family patterns and environmental degradation, as well as persistent inequalities and poverty, particularly in developing countries where the majority of older persons are already living.

To advance the movement for active ageing, all stakeholders will need to clarify and popularize the term "active ageing" through dialogue, discussion and debate in the political arena, the education sector, public fora and media such as radio and television programming.

Action on all three pillars of active ageing needs to be supported by knowledge development activities including evaluation, research and surveillance and the dissemination of research findings. The results of research need to be shared in clear language and accessible and practical formats with policy makers, nongovernmental organizations representing older people, the private sector and the public at large.
International agencies, countries and regions will need to work collaboratively to develop a relevant research agenda for active ageing.

WHO is committed to work in collaboration with other intergovernmental organizations, NGOs and the academic sector for the development of a global framew ork for research on ageing. Such a framework should reflect the priorities ex pressed in the International Plan of Action on Ageing 2002 and those in this document.

\section{Conclusion}

In this document, WHO offers a framework for action for policymakers. Together with the newly-adopted UN Plan of Action on Ageing, this framework provides a roadmap for designing multisectoral active ageing policies which will enhance health and participation among ageing populations while ensuring that older people have adequate security, protection and care when they require assistance.

WHO recognizes that public health involves a wide range of actions to improve the health of the population and that health goes beyond the provision of basic health services. Therefore, it is committed to work in cooperation with other intemational agencies and the United Nations itself to encourage the implementation of active ageing policies at global, regional and national levels. Due to the specialist nature of its work, WHO will provide 
technical advice and play a catalytic role for health development. However, this can only be done as a joint effort. Together, we must provide the evidence and demonstrate the effectiveness of the various proposed courses of action. Ultimately, however, it will be up to nations and local communities to develop culturally sensitive, gender-specific, realistic goals and targets, and implement policies and programmes tailored to their unique circumstances.
The active ageing approach provides a framework for the development of global, national and local strategies on population ageing. By pulling together the three pillars for action of health, participation and security, it offers a platform for consensus building that addresses the concerns of multiple sectors and all regions. Policy proposals and recommendations are of little use unless follow-up actions are put in place. The time to act is now. 


\section{References}

Aboderin I, Kalache A, Ben-Shlomo Y, Lynch JW, Yajnik CS, Kuh D, Yach D (2002). Life Course Perspectives on Coronary Heart Disease, Stroke and Diabetes: Key Issues and Implications for Policy and Research. Geneva: World Health Organization.

Action on Elder Abuse (AEA) (1995) Bulletin (11) MayJune. London

Botev N (1999). "Older persons in countries with economies in transitions." Population Ageing: Challenges for Policies and Programmes in Developed and Developing Countries. United Nations Population Fund and CBGS Population and Family Study Centre. New York: United Nations Population Fund.

Cutler D (2001). "Declining Disability Among The Elderly." Health Affairs Vol 20. (6):11-27

Dipollina L, Sabate E (2002) "Medication adherence to long term treatments in the elderly." In Sabate E. (ed). WHO Adherence Report: A review of the evidence, Geneva: World Health Organization. (forthcoming)

Doll R (1999) Risk from tobacco and potentials for health gain. International Journal of Tuberculosis and Lung Disease. 3 (2): 90-9

Gironda M and Lubben J (In press). "Preventing loneliness and isolation in older adulthood". In T Gullotta and M Bloom (Eds). Encyclopedia of Primary Prevention and Health Promotion. New York: Kluwer Academic/Plenum Publishers.

Gray MJA (1996) "Preventive Medicine”. in: Epidemiology in Old Age. Ebrahim S and Kalache A (eds) London: BMJ Publishing Group

Guralnick JM and Kaplan G (1989). "Predictors of healthy aging: prospective evidence from the Almeda County Study". American Journal of Public Health, 79: 703-8.

Gurwitz JH and Avorn J (1991). "The ambiguous relationship between aging and adverse drug reactions". Annals of Internal Medicine, 114: 956-66.

International Labour Office (ILO) (2000). "Income security and social protection in a changing world". World Labour Report. Geneva: ILO.
Jacobzone S and Oxley H (2002). "Ageing and Health Care Costs". International Politics and Society (1) http://www.fes.de/ipg/ONLINE2 2002/INDEXE.HTM

Jernigan DH, Monteiro M, Room R and Saxena S (2000). "Toward a global alcohol policy: alcohol, public health and the role of WHO". Bulletin of the World Health Organization, 78 (4), 491.

Kalache A and Keller I (2000). "The greying world: a challenge for the 21st century". Science Progress 83 (1), 33-54

Kalachea A and Kickbusch I (1997) "A global strategy for healthy ageing." World Health. (4) July-August, 4-5

Kirkwood T (1996) "Mechanisms of Ageing." In Epidemiology in Old Age. Ebrahim S and Kalache A (eds) London: BMJ Publishing Group

Lynch, JW, Smith GD, Kaplan GA, House JS (2000). "Income inequality and mortality: importance to health of individual income, psychosocial environment and material conditions". British Medical Journal, 320: 1200-04.

Manton K and Gu X (2001). "Changes in the prevalence of chronic disability in the United States, black and nonblack population above age 65 from 1982 to 1999. Proceedings of the National Academy of Sciences, 22: 6354-9.

Merz CN and Forrester JS (1997). "The secondary prevention of coronary heart disease". American Journal of Medicine, 102: 573-80.

Murray C and Lopez A (1996). The Global Burden of Disease. Oxford University Press.

OECD (1998). Maintaining Prosperity in an Ageing Society. Paris: Organization for Economic Cooperation and Development.

Pal J et al. (1974) "Deafness among the urban community - an epidemiological survey at Lucknow (U.P.)." Indian J Med Res 62; 857-868

Singer B and Manton K (1998). "The effects of health changes on projections of health service needs for the elderly population of the United States". Proceedings of the National Academy of Sciences, 23: 321-35. 
Smits CH, Deeg DM and Schmand B (1999). "Cognitive functioning and health as determinants of mortality in an older population". American Journal Epidemiology, 150 (9): 978-86.

Sugiswawa S, Liang J, Liu X (1994). "Social networks, social support and mortality among older people in Japan". Journals of Gerontology, 49: S3-13.

United Nations (UN) (2001). World Population Prospects: The 2000 Revision.

U.S. Centers for Disease Control (1999). Lower Direct Medical Costs Associated with Physical Activity.

Atlanta: CDC. See http://www.cdc.gov/nccdphp/dnpa/ pr-cost.htm

U.S Department of Health and Human Services (1999). An Ounce of Prevention ... What Are the Returns? Atlanta: U.S Department of Health and Human Services, Centers for Disease Control and Prevention.

U.S Preventive Services Task Force, (1996). Guide to Clinical Preventive Services, 2nd Edition. Baltimore: Williams and Wilkins.

WHO (1994). Statement developed by WHO Quality of Life Working Group. Published in the WHO Health Promotion Glossary 1998. WHO/HPR/HEP/ 98.1 Geneva: World Health Organization

WHO (1997). Global Elimination of Avoidable Blindness. WHO/PBL/97.61 Rev.2. Geneva: World Health Organization

WHO (1998) Growing Older. Staying Well. Ageing and Physical Activity in Everyday Life. Prepared by Heikkinen RL. Geneva: World Health Organization.

WHO (1998a). Life in the 21st Century: A Vision for All (World Health Report). Geneva: World Health Organization.

WHO (1999) World Health Report, Database. Geneva: World Health Organization.

WHO (2000). Global Forum for Health Research: The 10/90 Report on Health Research. Geneva: World Health Organization.

WHO (2000a). Health Systems: Improving Performance (World Health Report). Geneva: World Health Organization.
WHO (2000b). Home-Based and Long-term Care, Report of a WHO Study Group. WHO Technical Report Series 898. Geneva: World Health Organization.

WHO (2000c). Long-Term Care Laws in Five Developed Countries: A Review. WHO/NMH/CCL/00.2. Geneva: World Health Organization.

WHO (2001). Innovative Care for Chronic Conditions. Meeting Report, 30-31 May 2001, WHO/MNC/CCH/ 01.01. Geneva: World Health Organization.

WHO (2001a) Mental Health: New Understanding, New Hope (World Health Report). Geneva: World Health Organization.

WHO (2002) Developing and validating a methodology to examine the impact of HIV/AIDS on older caregivers - Zimbabwe case study. Geneva: World Health Organization. (in press)

WHO (2002a) Global Burden of Disease. Review. Geneva: World Health Organization. (forthcoming)

WHO/INPEA (2002). Missing Voices: Views of Older Persons on Elder Abuse. WHO/NMH/NPH/02.2 Geneva: World Health Organization

Wilkinson RG (1996). Unhealthy Societies: The Affliction of Inequality. London: Routledge.

Wilson DH et al. (1999) "The epidemiology of hearing impairment in the Australian adult population." Int J Epidemiol. 28:247-252

Wolf DA (2001) "Population change: friend or foe of the chronic care system" Health Affairs Vol.20 (6) 28-42

World Bank (1999). Curbing the Epidemic: Governments and the Economics of Tobacco Control. Washington: World Bank.

World Bank (2001). World Development Indicator Database, Washington: World Bank. http:// www.worldbank.org/data/wdi2001/pdfs/tab2_6.pdf

Yach D (1996) "Redefining the scope of public health beyond the year 2000". Current Issues in Public Health, 2: 247-252. 
We gratefully acknowledge the support provided by Health Canada. UNFPA contributed to the printing of the brochure through the Geneva International Network on Ageing (GINA).

Graphic Design: Marilyn Langfeld

(C) Copyright World Health Organization, 2002

This document is not a formal publication of the World Health Organization (WHO), and all rights are reserved by the Organization. The paper may, however, be freely reviewed, abstracted, reproduced and translated, in part or in whole, but not for sale nor for use in conjunction with commercial purposes.

The views expressed in documents by named authors are solely the responsibility of these authors. 

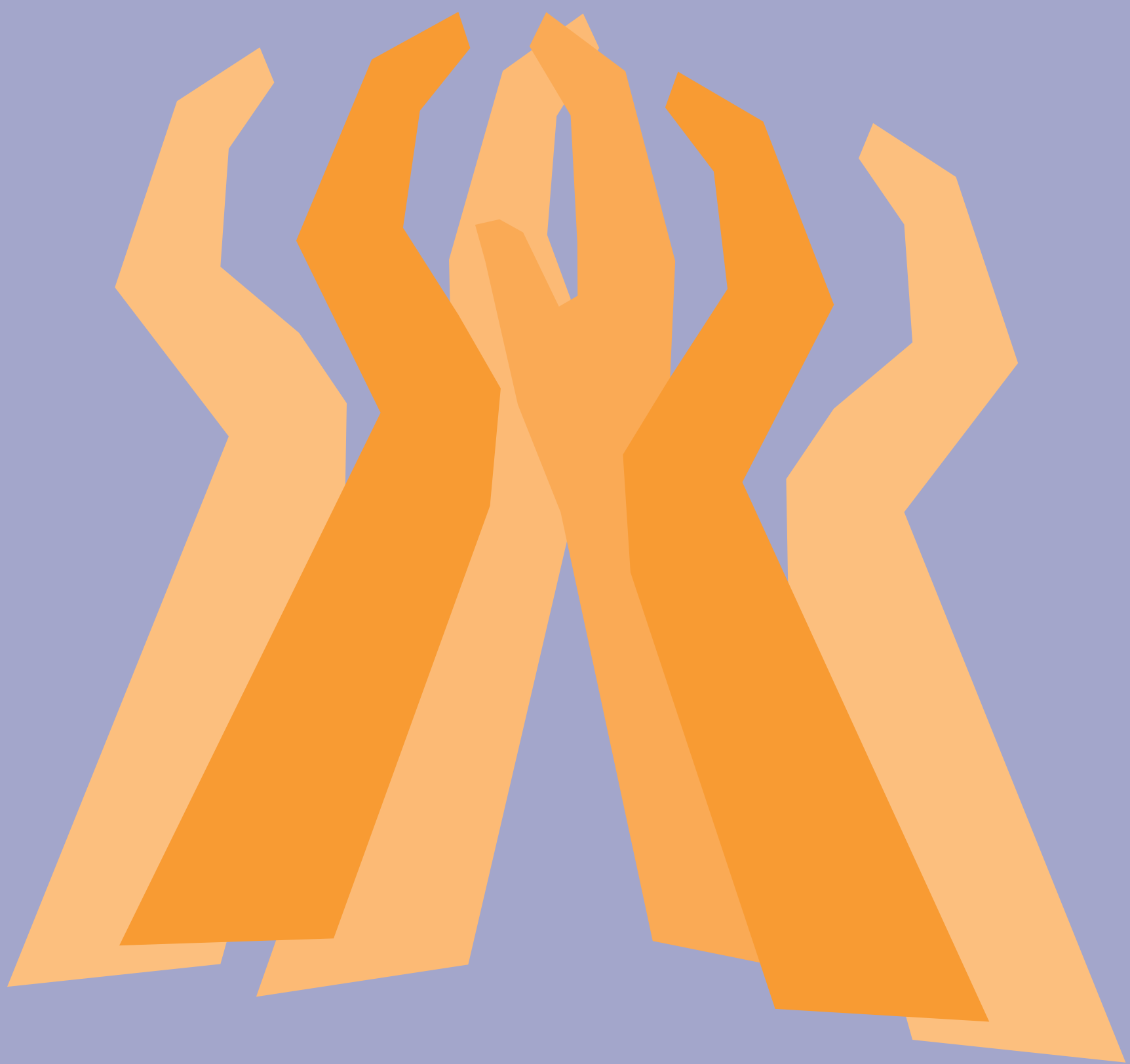

World Health Organization

Noncommunicable Disease Prevention and Health Promotion Ageing and Life Course

20 Avenue Appia, CH 1211 Geneva 27, Switzerland

Fax: +41-22-791 4839 Email: activeageing@who.int 